\title{
REGULATION, INNOVATION, AND THE INTRODUCTION OF NEW TELECOMMUNICATIONS SERVICES*
}

\author{
James E. Prieger \\ Department of Economics \\ University of California, Davis \\ One Shields Avenue \\ Davis, CA 95616-8578 \\ jeprieger@ucdavis.edu
}

November 7, 2000

\begin{abstract}
JEL codes: L51, L96

I examine the effects of FCC regulation on the innovation and introduction of advanced telecommunications services in the U.S. An interim of lighter regulation provides an "experiment" to test the regulatory regime's impact on innovation. The econometric model comprises an arrival process (for service innovation) followed by a duration process (for regulatory delay). The number of services the firms created during the interim is $60-99 \%$ higher than the model predicts they would have created if the stricter regulation had still been in place. Overall, firms would have introduced $62 \%$ more services to consumers during the study period if the regulation had not been in place.
\end{abstract}

\section{PRELIMINARY: DO NOT CITE WITHOUT PERMISSION}

\footnotetext{
${ }^{*}$ This work is based on chapter 5 of my dissertation (Prieger, 1999). I acknowledge the support of many people at Ameritech, LECG, Inc., and the University of California, Berkeley, without which this study would not have been possible. At Ameritech, I thank in particular Ken Dunmore and Larry Strickling (now at the FCC). At UC Berkeley, I thank my dissertation advisor Richard Gilbert and others. Early parts of the project received financial support from Ameritech through LECG; all opinions and errors are my own.
} 


\section{Introduction}

Innovation and new goods are important forces driving economic growth and increasing the welfare of consumers. In regulated industries such as telecommunications, however, firms attempting to introduce new services often must contend with restrictions designed more to protect the status quo than to promote innovation. Despite the prevalence of such restrictions, little effort has been devoted to measuring the effects of regulation on product innovation. Since Joskow and Rose (1989) noted with distress a decade ago that such studies were virtually non-existent, the dearth of empirical work on regulation and product innovation has largely remained. Notwithstanding, quantification of the impacts of regulation on innovation is vitally needed to inform public policy. This study examines the effect of a particular regulatory regime on the creation and introduction of new telecommunications services. My contribution is twofold. First, I quantify the effects of regulation in a real-world regime, uncovering the extent to which regulation reduces innovation and delays introduction of new services. Second, I develop an econometric model suitable for studying the introduction of regulated goods. The model comprises an arrival process for innovation followed by a correlated duration process for the regulatory delay that drives a wedge between innovation and introduction to consumers.

The literature looking at the effects of regulation on innovation focuses almost exclusively on cost reduction, or process innovation. Creating new goods-product innovation - has received scant attention. The only empirical study of which I am aware looking at product innovation and telecommunications regulation is Mueller (1993), who finds (non-econometric) evidence suggesting that deregulation was successful at speeding new service introductions in Nebraska. There are a few studies quantifying the impact of incentive regulation on process innovation. Taylor, Zarkadas and Zona (1992), Greenstein, McMaster and Spiller (1995), and Ai and Sappington (1998) all find that incentive regulation of various forms (price caps, earnings sharing, etc.) generally speeds the diffusion of telecommunications infrastructure compared with traditional rate of return regulation. 
Unlike these studies, which look at the means of providing a service, I look directly at the new services that consumers purchase.

The data for the study are from the Comparably Efficient Interconnection (CEI) regulation put on dominant telecommunications firms wishing to offer information services. The Bell Operating Companies $^{1}$ (BOCs) and AT\&T have introduced enhanced services integrated with their networks (such as protocol conversion, voice mail, and audiotext information services) on a restricted basis since 1987. These services require access to the local telephone network to function. For example, voice mail service for a household must intercept and reroute incoming calls. Because the carriers were the sole suppliers of the necessary network elements, the Federal Communications Commission (FCC) required assurance - via a lengthy approval process - that they were not taking advantage of their monopoly position. The approval process begins when the carrier submits a plan to the FCC offering competitors "comparably efficient interconnection" to the network. The CEI plan theoretically allows rivals to offer similar services, although the firms complain that the process merely raises the cost of introducing a service. For an interim around 1992-1995 such detailed plans and time-consuming approvals were not required. During that time, the carriers promised to follow FCC-approved open network plans, and the FCC allowed them to introduce new enhanced services with no special oversight. Section 2 describes the CEI regime in more detail.

Carriers likely found it more attractive to introduce new services in the interim of lighter regulation (hereafter the "interim"). The CEI requirements reduced the expected profit of introducing a new service for at least three reasons. First, there are substantial direct costs of preparing a CEI plan, which require great amounts of technical and legal staff time. One BOC claims that the CEI plan requirement is "the most significant" regulatory burden imposed on BOCs' enhanced service operations. ${ }^{2}$ Second, the plan reveals information to potential competitors before the service is introduced, allowing competitors to preempt the BOCs. ${ }^{3}$ Third, there are long delays associated with plan approval - over 200 days on average, a long time in the rapidly advanced telecommunications industry. ${ }^{4}$ Once proposed, the plans typically went through several rounds of public comment and 
rebuttals, and in six cases the FCC requested changes to the plans. The delay reduces the present value of a proposed new service and allows competitors to beat the regulated firm to market. The econometric model for innovation and introduction is presented in sections 3 and 4.

To see if the release from regulation spurred more innovation, I estimate a prediction interval (PI) for the interim using data from the regulated periods (section 5.1). The actual number of new services created during the interim lies outside the "regulated-conditions" PI, evidence that the behavior of the firm changed when released from the regulation. An alternative approach (one typically followed in regulation studies) would be to use the data from both the regulated and unregulated periods and to include a dummy variable (DV) for the interim. I choose the PI methodology for three reasons, given in increasing order of importance. First, I have more complete data for the regulated periods. During the interim I know only the total count of new services, not when each occurred. ${ }^{5}$ Second, unlike a DV, the PI approach does not constrain any coefficients or even the parametric form of the model to be equal between the regulated and unregulated periods. I place no restrictions on how the behavior of the firm might change. Finally, the PI approach sets a higher hurdle for the data to cross before we accept evidence of structural change. For example, under the DV approach, one might find that the coefficient for the interim is statistically significant but numerically small, so that the mean counts from the two periods are close. Thus counts generated from the two periods will be nearly indistinguishable and the evidence for structural change will not be convincing. The PI approach requires that the observed count from the interim have a small probability of being generated from the regulated process-a stricter test of structural change.

The analysis in section 5.1 bears out that some otherwise profitable services are not financially viable under the CEI regime. The number of services the firms created during the interim is 60-99\% higher than the model predicts they would have created if the stricter regulation had still been in place. The smaller predictions are from models with fixed effects only; the larger predictions control for covariates. Omitted variables tests indicate that the increase in services is 
not driven solely by demand, technological factors, or competition. In fact, controlling for these factors strengthens the conclusion that innovation increased during the interim. A semiparametric technique corroborates the prediction from the parametric models. In section 5.2, I also look at the magnitude and determinants of product introduction delay caused by the regulatory review process. The FCC is slower approving plans that are more complex, and approves second adopters' plans $41 \%$ quicker than pioneers' plans.

There also appear to be direct and indirect interactions in the model between innovation and regulatory delay. The direct effects include the finding that as expected regulatory delay increases, the firms innovate less. The direct effects in the regulatory delay model go the other direction: the more services in the regulatory inbox, the quicker approval is granted. The direct effects are not

highly statistically significant, so the conclusions remain tentative here. The indirect effects show up through positive estimated correlation between the innovation and regulatory delay parts of the model (net of the direct effects), possibly evidence of unobserved factors that cause both more innovation and longer delays.

Using the estimated model to simulate the innovation and introduction process with and without the CEI regulation and regulatory delay, I find that firms would have introduced $62 \%$ more services to consumers during the study period if the regulation had not been in place (section 6). This projection exercise requires stronger assumptions than does the prediction interval exercise in section 5.1, because it assumes a parametric form for innovation during the unregulated interim.

\section{The Comparably Efficient Interconnection Regime}

Before 1986, the regulated telecommunications carriers were under a requirement of structural separation: AT\&T or any BOC wishing to offer enhanced services was required to form a separate subsidiary. The separation rules erected a wall between the monopoly segment of the industry and the competitive segment, of which enhanced services were a part. Through the Computer III series 
of orders (1986-1989), the FCC allowed integrated provision of enhanced services. ${ }^{6}$

Computer III established a long-term goal, Open Network Architecture (ONA), and a shortterm plan, CEI, to open the dominant firms' networks to competitors. ${ }^{7}$ To gain the FCC's approval to offer enhanced services, a carrier had to fulfill two requirements. First, for each service it had to develop a CEI plan to abide by certain safeguards. The CEI plan allowed enhanced service providers (ESPs) comparable interconnection to the elements of the network used by the particular enhanced service. Each proposed service necessitated a new CEI plan. Second, the carrier had to develop a longer range ONA plan to open up the rest of their network by offering all the individual "building blocks" of the network to all customers. The FCC stated that after a carrier's ONA plan was approved, the requirement of structural separations would be lifted altogether, and individual CEI plans would no longer be required.

The BOCs began to submit CEI plans to the FCC around the time the ban on gateway services was removed in September $1987 .{ }^{8}$ In June 1990, a court case (California I) vacated the Computer III orders, requiring the FCC to disallow development and introduction of new enhanced services (although carriers were allowed to continue to offer existing advanced services and to file for CEI waivers). The FCC strengthened the safeguards criticized in the California I decision and resumed the CEI regime in February 1992. In 1992 and 1993, the BOCs individually received final approval of their ONA plans and a waiver of all structural separations requirements, which granted the freedom to offer enhanced services without filing CEI plans.

In October 1994, another court case, California III, effectively forced the FCC to reinstate the CEI plan requirements. The FCC allowed the BOCs to continue to provide existing enhanced services on an integrated basis. However, the BOCs were required to file retroactive CEI plans for services introduced after the lifting of structural separation. The retroactive CEI filings in January 1995 thus provide an enumeration of new services during the remand period. Thereafter, the CEI plan requirement remained in effect for the BOCs until February 1999. In February 1999 the FCC eliminated filing and pre-approval of CEI plans, instead requiring only that the BOCs post CEI 
plans on the Internet for new or modified enhanced services. ${ }^{9}$ To avoid contamination of the data by anticipation effects, I use data only through 1997 for the innovation estimations.

Figure 1 depicts the observability of innovation in the data from the public record during the CEI regime. The time line shows when enhanced services were being developed and introduced by carriers and the activity is observable through CEI filings (the black areas), when services were being developed and introduced but the activity is not observable in the public record until a later date (the gray areas), and when development and introduction are disallowed (the white areas). The extent to which the firms anticipated these regime changes affects the interpretation of the data; I explore this issue in section 6.

\section{A Model for Regulated Service Innovation and Introduction}

In the course of introducing regulated telecommunications services to subscribers, a firm goes through two steps. The firm first creates a new end-user service (possibly using technology developed by other firms); I refer to this as innovation. After innovation, the services are not introduced to subscribers until they are approved by the regulator. In this paper innovation will always refer to the point in time at which the firm has created the service; introduction will always refer to the point at which the regulator has approved the service and allows the firm to offer it to subscribers. Modeling this progression requires a statistical framework that incorporates both steps; I use an arrival process followed by a duration process. The arrival process generates the new services, and the duration process determines the delay between innovation and introduction to consumers.

Merely applying a count model to new service introductions (i.e., the outcome of the second step) would potentially bias inference about the impact of regulation. To be concrete, consider the following example. Suppose in the data the number of new service introductions increases after a regulatory regime change (the case in the present data). Without examining the innovation and introduction processes distinctly, one could not distinguish two competing hypotheses: 1) 
innovation has not changed but regulatory delay has decreased; and 2) innovation has increased. In the former case, the increase in the number of new services reflects the emptying out of the regulatory queue and is a transient phenomenon. In the latter case, the increase in new services may reflect a longer-lasting change in the firms' behavior. The model I propose separates the innovation and the delay processes and would detect (given enough data) any changes in either innovation or regulatory delay.

The model presented in the next section is a generalized Poisson arrival process followed by a generalized Weibull duration process. In particular, arrivals (innovation) follow a Poisson event process. After arrival from the population of potential new services, a new service immediately enters a Weibull duration process that determines the length of the time until regulatory approval (see Figure 2). The hazard rate of the Weibull process is determined from time-varying covariates. The conditional means of the distributions are modeled as exponential functions of economic, demographic, and regulatory explanatory panel data.

The model is formally equivalent to an infinite-server queuing model. ${ }^{10}$ The equivalence of the model to a queuing system allows adaptation of a result from the queuing literature for the projection exercise carried out in Section 6.

\section{The Econometric Implementation}

In this section I construct the likelihood of the data from the arrival and regulatory approval processes, from which maximum likelihood (ML) parameter estimates may be obtained. The idea of the model is simple, although the details require some care because the count and duration observations do not match one for one as would be the case in most bivariate distributions. The innovation of services generates count data. Regulatory delay time of each service generates duration data. A bivariate unobserved heterogeneity term admits correlation between the counts and the durations. The likelihood for the data is first found conditional on the heterogeneity 
terms. The unobserved heterogeneity terms are then integrated out, resulting in the unconditional likelihood. The model nests several simpler models, including independent standard Poisson and Weibull models, thus allowing specification testing.

To construct the likelihood, the marginal distributions and the nature of the correlation between the count and duration processes must be specified. Let the number of arrivals in period $t, t=$ $1, \ldots, T$, from firm $k \in K$ and of type $\ell \in L$, be $n_{t k \ell}$, a realization of a count (non-negative integer) valued random variable. In the data, $K \equiv\{1, \ldots, 8\}$ represents AT\&T and the seven Bell Operating Companies, and $L \equiv\{1,2,3\}$ represents the type of CEI filing (plans, amendments, and waivers); descriptions of the filing types are below in the data description section. The length of period $t, s_{t}>0$, is usually 0.25 (one quarter year) but may be less if the quarter is incompletely observed. Denote by $f\left(n_{t k \ell} \mid u_{1 t}\right)$ the probability density function (pdf) of $n_{t k \ell}$, conditional on firm and type fixed effects $\alpha_{1 k}$ and $\alpha_{2 \ell}$, covariates $\mathbf{x}_{t}$, coefficient vector $\boldsymbol{\beta}$, and a random effect $u_{1 t}$ (the notation suppresses explicit dependence on $\alpha_{1 k}, \alpha_{2 \ell}, \mathbf{x}_{t}$, and $\boldsymbol{\beta}$ for simplicity). The simplest such count process is the Poisson model with rate and pdf

$$
\begin{aligned}
& \lambda_{t k \ell}=\exp \left(\alpha_{1 k}+\alpha_{2 \ell}+\mathbf{x}_{t}^{\prime} \boldsymbol{\beta}+u_{1 t}\right) \equiv \lambda_{t k \ell}^{0} \exp \left(u_{1 t}\right) \\
& f\left(n_{t k \ell} \mid u_{1 t}\right)=\exp \left(-s_{t} \lambda_{t k \ell}+n_{t k \ell} \log \left(s_{t} \lambda_{t k \ell}\right)\right) / n_{t k \ell} !
\end{aligned}
$$

respectively. The random effect $u_{1 t}$, for simplicity taken to be common across firms and types within a period, is an unobserved heterogeneity term with variance $\tau_{1}^{2} \geq 0$. Assume that $E\left(e^{u_{1 t}}\right)=1$, so that $E\left(n_{t k \ell}\right)=s_{t} \lambda_{t k \ell}^{0}=s_{t} \exp \left(\alpha_{1 k}+\alpha_{2 \ell}+\mathbf{x}_{t}^{\prime} \boldsymbol{\beta}\right)$. The inclusion of $u_{1 t}$ results in a generalized Poisson model that relaxes the equality of the mean and the variance implied by the simple Poisson model and allows overdispersion (Cameron and Trivedi, 1998). ${ }^{11}$ The random effect also allows correlation with the duration part of the model, as explained below.

Associated with each counted event (innovation) is a duration (regulatory delay). The durations are assumed to follow a Weibull distribution with a hazard that depends on time-varying covariates and unobserved heterogeneity terms. The notation threatens to become cumbersome at this point 
because the number of durations does not match the number of periods (instead it matches the sum of all the counts) and because the durations may begin in one period and end in another. I term a straddling duration to be one that begins in one period and ends in another. To construct the likelihood of this part of the model, set aside the heterogeneity and the period-matching problem for the moment and focus on time-varying covariates and straddling for a single duration.

Let $y$ be the realization of the random duration variable $Y>0$. Let vector $\mathbf{Z}(\varsigma)$ be the value at time $\varsigma$ of explanatory variables that are exogenous in the sense of Lancaster (1990, p.28), and let $\mathbf{Z}(a, b)$ be the time-path of $\mathbf{Z}$ on $[a, b]$. The hazard rate of $Y$ at time $\varsigma$ (conditional on $\mathbf{Z}(\varsigma)$ and a finite parameter vector $\boldsymbol{\theta})$ is:

$$
h(\varsigma ; \boldsymbol{\theta}, \mathbf{Z}(\varsigma)) \equiv \lim _{d \varsigma \rightarrow 0} \operatorname{Pr}[\varsigma \leq Y<\varsigma+d \varsigma \mid Y \geq \varsigma, \boldsymbol{\theta}, \mathbf{Z}(\varsigma, \varsigma+d \varsigma)]
$$

(Lancaster, 1990). Note that only the contemporaneous value of $\mathbf{Z}$ enters $h$, not its entire path. In the present application the covariates are constant within each period $t$ but may change between periods; thus $\mathbf{Z}$ is a step function with jumps at the times corresponding to the calendar dates of the beginnings of the periods. Label these points in $[0, y]$, endpoints included, as $\left(y_{0}, y_{1}, \ldots, y_{J}\right)$, and let $h_{j}(\varsigma ; \boldsymbol{\theta})$ be the hazard rate in period $j$ before the $j$ th jump (set $h_{J}$ equal to the hazard on $\left.\left[y_{J-1}, y_{J}\right]\right)$. A single straddling duration has pdf:

$$
f(y, \mathbf{Z}(0, y))=h_{J}(y) \exp \left(-\sum_{j=1}^{J} \int_{y_{j-1}}^{y_{j}} h_{j}(\varsigma) d \varsigma\right)
$$

(see Peterson (1986)), where the dependence on $\boldsymbol{\theta}$ is suppressed in the notation. Define the "completing spell" indicator $d_{j}$, where $d_{j}=1$ if duration $y$ is observed completed after length $y_{j}$ and 0 if not. When duration $y$ is observed in its entirety, then $d_{j}=0$ for all but the final $d_{J+1}$. The final $d_{J}$ will also be 0 if duration $y$ is censored (e.g., ongoing regulatory delay spells at the end of the observation period). Define

$$
g_{j}\left(y_{j}, y_{j-1}, d_{j}, \mathbf{Z}\left(y_{j-1}, y_{j}\right)\right)=\left\{\begin{array}{l}
\frac{1-F_{j}\left(y_{j}\right)}{1-F_{j}\left(y_{j-1}\right)} \text { if } d_{j}=0 \\
\frac{f_{j}\left(y_{j}\right)}{1-F_{j}\left(y_{j-1}\right)} \text { if } d_{j}=1
\end{array}\right.
$$


where $F_{j}$ and $f_{j}$ represent the Weibull cumulative density function (cdf) and pdf, respectively, under hazard $h_{j}$ and conditional on $\mathbf{Z}\left(y_{j-1}, y_{j}\right)$. Then after some algebra equation (4) can be rewritten as

$$
f(y, \mathbf{Z}(0, y))=\prod_{j=1}^{J} g_{j}\left(y_{j}, y_{j-1}, d_{j}, \mathbf{Z}\left(y_{j-1}, y_{j}\right)\right) .
$$

For the Weibull model, $f_{j}$ and $F_{j}$ take the form

$$
\begin{gathered}
f_{j}(y)=\frac{1}{y \sigma}\left(\frac{y}{\mu_{j}}\right)^{\frac{1}{\sigma}-1} \exp \left(-\left[\left(\frac{y}{\mu_{j}}\right)^{\frac{1}{\sigma}}\right]\right) \\
F_{j}(y)=1-\exp \left(-\left[\left(\frac{y}{\mu_{j}}\right)^{\frac{1}{\sigma}}\right]\right),
\end{gathered}
$$

where the shape parameter $\sigma$ (common to all periods and observations) and the rate parameter $\mu_{j}$ are both positive. The Weibull mean is $\sigma \Gamma(\sigma) \mu_{j}$. The Weibull model allows duration dependence; i.e., a nonautonomous hazard rate. Values of $\sigma$ less than one (underdispersion) yield a hazard rate that increases over the duration; this is known as positive duration dependence (the likelihood of the spell ending in the next instant increases as time goes on). Values of $\sigma$ greater than one (overdispersion) yield a decreasing hazard rate and negative duration dependence.

Now we can deal with the period-matching problem. Consider the entire sample of $M \equiv$ $\sum_{t=1}^{T} \sum_{k \in K} \sum_{\ell \in L} n_{t k \ell}$ durations, where each straddling duration is counted only once. Let the $m$ th observation have $J_{m}$ terms after splitting at the period changes, resulting in a split sample $\left(\left(y_{m j}\right)_{j=1}^{J_{m}}\right)_{m=1}^{M}$. For simplicity of notation, relabel these with the single index $i$, resulting in $\left(y_{i}\right)_{i=1}^{N}$, where $N \equiv \sum_{m=1}^{M} J_{m}$, preserving the order of the series for the sake of the arguments of $g$ in (5). Similarly, relabel the associated completing spell indicators $d_{i}$, rate parameters $\mu_{i}$, likelihood terms $g_{i}$, and explanatory variables $\mathbf{z}_{i}$ (the appropriate levels of $\mathbf{Z}(\varsigma)$ ), $i=1, \ldots, N$. Note that $\mathbf{z}_{i}$ is a vector of constants. To match the split durations to the periods associate each $i$ with an index set $I_{t}$ so that $\left\{i \mid i \in I_{t}\right\}$ are the indices pertaining to period $t$.

With the new notation we can introduce the heterogeneity. For $i \in I_{t}$, the rate parameter $\mu_{i}$ is 
modeled as

$$
\mu_{i}=\exp \left(\gamma_{1 k}+\gamma_{2 \ell}+\mathbf{z}_{i}^{\prime} \boldsymbol{\delta}+u_{2 t}\right)=e^{u_{2 t}} \exp \left(x_{i}^{\prime} \beta\right)
$$

where $\gamma_{1 k}$ and $\gamma_{2 \ell}$ are firm and filing type fixed effects as in the count model. Thus $\boldsymbol{\theta}$ from (3) is $(\boldsymbol{\gamma}, \boldsymbol{\delta}, \sigma)$. Parallel to the count model above, the random effect $u_{2 t}$ is common across firms, types, and all split durations within a period; it is an unobserved heterogeneity term with variance $\tau_{2}^{2} \geq 0$. Assume that $E\left(e^{u_{2 t}}\right)=1$. The inclusion of $u_{2 t}$ results in a mixture model (Lancaster, 1990, ch.4) that generalizes the standard Weibull model and allows correlation with the duration part of the model.

Taken together, $\left(u_{1 t}, u_{2 t}\right)$ represent unobserved period-specific heterogeneity. To complete the model and to specify the correlation between the counts and durations, let $\left(u_{1 t}, u_{2 t}\right)$ be defined by

$$
\begin{aligned}
& u_{1 t}=\tau_{1}\left(\eta_{1 t}-\tau_{1} / 2\right) \\
& u_{2 t}=\tau_{2}\left(\rho \eta_{1 t}+\sqrt{1-\rho^{2}} \eta_{2 t}-\tau_{2} / 2\right)
\end{aligned}
$$

where $\left(\eta_{1 t}, \eta_{2 t}\right)$ are iid draws from a bivariate standard normal distribution, $\tau_{1}, \tau_{2} \geq 0$, and $\rho \in[-1,1]$. Then $\left(u_{1 t}, u_{2 t}\right)$ are bivariate normal with variance $\left(\tau_{1}^{2}, \tau_{2}^{2}\right)$ and correlation $\rho$. Thus $\rho$ is the key parameter governing correlation between the count and regulatory delay processes. If $\rho$ is positive, then departures from the means in the count and regulatory delay processes are positively correlated, as might happen if there is "regulatory congestion" due to the finite resources of the regulator (beyond that which may be captured by included covariates). If $\rho$ is negative, then the count and regulatory delay processes are negatively correlated, as might happen if the regulator feels pressure to expedite service approvals when there are many new services created or if the firm submits more new services to the regulator when approval times are short. The definition of $\left(u_{1 t}, u_{2 t}\right)$ ensures that $E\left(e^{u_{1 t}}\right)=E\left(e^{u_{2 t}}\right)=1$, as required above.

The joint pdf for the data in period $t,\left\{n_{t k \ell}, y_{i} \mid i \in I_{t}, k \in K, \ell \in L\right\}$, conditional on $\left\{y_{i-1}, u_{1 t}, u_{2 t} \mid i \in\right.$ 
$\left.I_{t}\right\}$, is then

$$
f\left(\left\{n_{t k \ell}, y_{i}\right\} \mid u_{1 t}, u_{2 t}\right)=\left[\prod_{k \in K} \prod_{i \in L} f\left(n_{t k \ell} \mid u_{1 t}\right)\right] \cdot\left[\prod_{i \in I_{t}} g_{i}\left(y_{i}, y_{i-1}, d_{i}, \mathbf{z}_{i} \mid n_{t k \ell}, y_{i-1}, u_{2 t}\right)\right]
$$

where the dependence on the parameters is suppressed in the notation. In the expression above, the form of $f\left(n_{t k \ell} \mid u_{1 t}\right)$, the Poisson pdf, is given by (2). The form of $g_{i}$, the contribution of split duration $i$ to the joint likelihood, is given by (5), (7), and (8). Note that $g_{i}$ is conditional on $n_{t k \ell}$, so that functions of current-period counts may be included in $\mathbf{z}_{i} \cdot{ }^{12}$ Current period durations are not allowed in $\mathbf{x}_{t}$ (although functions of past durations are allowed).

Since $\left(u_{1 t}, u_{2 t}\right)$ are not observed, one finds the unconditional joint pdf by integrating out $\left(u_{1 t}, u_{2 t}\right)$ :

$$
f\left(\left\{n_{t k \ell}, y_{i}\right\}\right)=E_{u_{1 t}, u_{2 t}} f\left(\left\{n_{t k \ell}, y_{i}\right\} \mid u_{1 t}, u_{2 t}\right)
$$

where $E_{u_{1 t}, u_{2 t}}$ denotes expectation taken over $\left(u_{1 t}, u_{2 t}\right)$. The log likelihood for all time periods is

$$
\ell\left(\boldsymbol{\alpha}, \boldsymbol{\beta}, \boldsymbol{\gamma}, \boldsymbol{\delta}, \rho, \sigma, \tau_{1}, \tau_{2}\right)=\sum_{t=1}^{T} \log f\left(\left\{n_{t k \ell}, y_{i}\right\}\right)
$$

The expectation in (11) is a double integral that cannot be solved analytically, requiring numerical methods. ${ }^{13}$ Estimation of this model therefore can be expensive when there are many covariates.

Although the general model (12) is rather complicated, it is about the simplest model that allows correlation between the innovation and regulatory delay processes. The model contains several familiar models as special cases. When $\rho=0$, the count and duration models are independent and may be estimated separately with full efficiency. Estimation of the independent models is numerically easier than of the joint model because the integrals in (11) are unidimensional. When $\tau_{1}=0$, the count model is the standard Poisson model with no accounting for overdispersion. When $\tau_{1}>0$ the count model is the generalized Poisson model. When $\rho=0$, these count models are independent of the regulatory approval process, and $(\boldsymbol{\alpha}, \boldsymbol{\beta})$ can be estimated consistently in either model by MLE based on (2) with $u_{1}$ identically equal to zero. ${ }^{14}$ Similarly, the duration model when $\rho=0$ (no correlation with the arrival process) may be estimated by MLE with the 
duration data. When $\tau_{2}=0$, the duration model is the standard homogeneous Weibull model. When, in addition, $\sigma=1$, the duration model is exponential. Estimating these restricted models may be useful for hypothesis testing and to provide starting values for estimation of the full model.

\section{Data and Estimation of the Model}

Since the innovation and regulatory delay models are independent if $\rho=0$, I first discuss each separately in sections 5.1 and 5.2. In section 5.3 I discuss the results from joint estimation of the fully correlated model.

\subsection{The Innovation Model}

\subsubsection{Data}

Data on all CEI plan filings and waiver requests from 1987 through 1997 were collected from the FCC Record (see the Appendix). The CEI plans, plan amendments, and waivers provide a record of new enhanced services that AT\&T and the BOCs wished to introduce on an unseparated basis during that time. Plans are the firm's proposal to meet the CEI safeguards for a new information service. Amendments are modifications to existing plans that are required when significant new functionality is added to a service. Waivers are requests for permission to offer a new service without meeting all of the required CEI safeguards, usually because of technological limitations. During the interim around 1993 to 1995, when CEI plans were not required, there is no way to track the introduction date of new services. However, after the Computer III remand, CEI plans for all services introduced during the interim were filed en masse, so one can count new services in retrospect. After the interim, the BOCs again filed CEI plans as new or amended services were introduced. Since each enhanced service requires either a CEI plan or a waiver, this data set encompasses all enhanced services offered by AT\&T and the BOCs.

To use the CEI plan filings as evidence of new service creation requires care. I deemed an 
amended plan to represent a new service only if it introduced new features or functionality and if it was not mandated by the FCC. ${ }^{15}$ The new services are summarized in Table 2. In all, 74 new enhanced services were introduced via CEI plans or waivers, and 27 were introduced during the interim, for a total of 101 services during the observation period.

To apply the methodology developed in Section 4, I first create the period-firm-type counts $\left(n_{t k \ell}\right)$, where the periods are the quarters between the start of 1987 and the end of 1997 that at least partially overlap with the "innovation observed" part of the timeline in Figure 1. The CEI filing types are plans, amendments, and waivers. Separating the filings by carrier and filing type this way allows the arrival process to differ among groups through the fixed effects represented by $\boldsymbol{\alpha}$ in (1). For example, services represented by Ameritech's CEI plans may be created at a different rate than services represented by PacBell's CEI waivers. The fixed effects are estimated by two sets of dummy variables; AT\&T is the excluded carrier in the firm dummies and plans are the excluded filing type dummy. Because the observations come from two disjoint periods (before and after the interim), I allow the mean of the duration process to differ during the latter period by including a dummy for the remand period (1995-1997).

\subsubsection{Estimation and Prediction}

The approach here is to fit the model to the service innovation data from the periods when the CEI regime was in place. Then, I can compare the model's prediction for the number of services introduced during the interim with the actual number of services introduced. If I find that many more services were introduced during the interim under the loosened regime than the model predicts, then the difference is evidence supporting the hypothesis that the lifting of the CEI regime spurred the introduction of new services.

The results of the simple Poisson model (i.e., $\tau_{1}=0$ ) estimation with only the firm, type, and remand fixed effects are in Table 4, column one. Positive coefficients increase the number of arrivals, ceteris paribus. The BOC fixed effects are all negative, meaning that the BOCs introduced services 
at a relatively slower rate than AT\&T (which matches the data in Table 2). The coefficients for amendments and waivers are also negative, implying that they were less frequent than plans. The indicator for the remand period is not significant, which indicates that the pace of innovation was comparable in the two regulated periods.

Now that I have characterized the BOCs' new service creation process under regulation, I can use the fitted model to answer the following question. If the lifting of structural separation requirements had not occurred and the BOCs were required to continue to submit CEI plans for new services, how many would I expect them to have submitted during the interim? If in fact the reduced burden of regulation during the interim accelerated the introduction of new services, then we should expect that my prediction will be significantly lower than the actual number of services introduced. This is indeed the case.

The predictor is based on the predicted yearly mean of the arrival process, $\hat{\lambda}_{t k \ell}^{0}$ from (1). ${ }^{16}$ To calculate the predicted number of new services from the BOCs during the interim, calculate $\hat{\lambda}_{t k \ell}^{0}$ using the estimated coefficients, multiply by the length of the interim for firm $k$, and sum over $k$ and $\ell .^{17}$ Note that the prediction for a BOC and plan type does not vary year to year in the fixed effects model, because it includes no varying regressors. It is a feature of the homogeneous Poisson model that such a predictor will reproduce the means in the raw data. What this version of the model allows that the raw data do not is a way to estimate the prediction variance.

The results of the prediction for each firm are in Table 5; the resulting total prediction, prediction interval, and $p$-value is in the first row of Table $6 .{ }^{18}$ The third column of Table 5 has the predicted number of new services we would have expected to see during the interim under the counter-factual assumption that the CEI regime was still in place. The interim was between 1.5 and 2.6 years long, differing for each firm because the FCC released them from the CEI requirements on different dates. If the structural separations requirements had not been lifted, we would expect to have seen about 16.3 new plans and amendments introduced by the BOCs during the interim. In fact, we saw 26, about $60 \%$ more than expected and outside the $95 \%$ prediction interval (see 
Table 6 ). The $p$-value of 26 services is 0.02 ; thus it is highly likely that the incentives to innovate did change during the interim. The predictors from the other models I estimate in the next section bolster this conclusion.

\subsubsection{Specification Checking}

Before I put much weight on the conclusions from the prediction exercise, it is important to test the baseline model for departures from the assumptions that it requires. Note that the nature of the observation period already avoids one of the common pitfalls of applied regulation studies. Typically, a change in regulation is a "one way street," with a deregulated period generating the second half of the observations. Many factors (such as competition) might lead to increased innovation over time, and such "before-and-after" studies typically cannot separate the effects of the regulation from secular trends in innovation (Sappington and Weisman, 1996). Because the interim occurs in the middle of my observation period, my study does not suffer from the same difficulty.

In this section I perform specification testing of several forms. Consider first the possibility that there is unobserved heterogeneity (i.e., that $\tau_{1}>0$ ). The second column of Table 4 differs from the previous fixed effects estimation only in that $\tau_{1}$ ) is a free parameter. The coefficient estimates do not change much (as expected; even if there is heterogeneity the simple Poisson model is consistent, although inefficient). In this estimation (and in the following two generalized Poisson

estimations) $\tau_{1}$ differs significantly from zero. ${ }^{19}$ Given that there is evidence of heterogeneity, I re-do the prediction exercise with the heterogeneous fixed effect model to make sure that the prediction interval still excludes 26 services (the actual number introduced) after incorporating the additional variance. The resulting prediction, prediction interval, and $p$-value is in the second row of Table 6. Twenty-six services still has a low $p$-value (0.02), providing corroboration for the earlier evidence that innovation changed during the interim.

Next consider the possibility that the mean is incorrectly specified through the omission of 
relevant variables. The first variable to check is expected regulatory delay time. As noted in the introduction, the long delays between innovation and introduction may be one reason that the regulation hampered innovation. An expected regulatory delay variable was constructed based on past realizations of regulatory delay (information that is common to all firms). The variable is calculated assuming firms update their expectation of regulatory delay using Bayes' rule as new information from recent past experience is received. ${ }^{20}$ Expected regulatory delay is added to the next two estimations: the congestion and economic variables estimations in Table 4 . In both estimations, the coefficient on log expected delay is negative-longer expected delay leads to less innovation. At sample averages, the elasticity of -0.33 in the congestion model implies that if expected delay were to rise by 152 days, one fewer service would be added to the total yearly innovation (all firms and plan types). ${ }^{21}$ The same calculation based on the economic variables elasticity of -0.60 shows that there would be one fewer service created if expected delay rose by only 83 days. Conversely, if regulatory delay were eliminated altogether, there would be from three (congestion) to six (economic variables) new services per year. The congestion effect is statistically significant only in the second estimation, so the conclusions here must remain tentative.

The next variables to check are economic and demographic variables that might affect the demand for new services. The economic variables estimation in Table 4 includes measures of consumer income (log per-capita real income in the BOC's territory) and BOC market size (log number of access lines) (all variables not also in column one are demeaned). Since AT\&T does not have a territory like the BOCs, AT\&T's observations must be dropped for this estimation. ${ }^{22}$ These variables are not significant, ${ }^{23}$ although access lines does have the expected sign (more lines increases new services).

Next I check controls for the changing size of the population of potential new services. I include the log of contemporaneous real R\&D spending by the carriers and by the whole U.S. telecommunications industry. The estimated coefficients in the economic variables model are statistically insignificant. ${ }^{24}$ The coefficient on firm R\&D, 0.275, implies that (at sample averages) about $\$ 50 \mathrm{M}$ 
above average $R \& D$ spending would generate a new service.

Competition to the BOCs might also influence service creation, although the effect could go either way. ${ }^{25}$ The first control for competition is the number of competitive access providers (CAPs) in the firm's territory. ${ }^{26}$ CAPs offer access services to high-volume customers in urban areas, and sometimes offer information services as well. The other control is more general: the contribution of the communications industry (net of the BOCs contribution) to real gross state product within the BOC's territory. ${ }^{27}$ This variable captures a broader range of competitive activity that the BOCs face than does the CAP variable. The estimated coefficients on both variables are negative, but neither coefficient is significant. ${ }^{28}$

A linear time trend, to capture any other omitted variable that grows over time (such as competitive pressure or the expected profitability of introducing new services), is also insignificant. Finally, a test of the joint hypothesis that all of the coefficients in the economic variables estimation that are not in the congestion estimation are zero fails to reject. ${ }^{29}$

Viewed as a whole, the evidence strongly indicates that omitted variables are not a problem for the fixed effects specification. Nevertheless, I re-do the prediction exercise based on the congestion and economic variables models; the results are in rows three and four of Table 6 . The predictors are calculated using the actual values of the covariates during the interim period (and therefore do not merely reproduce the sample mean of the dependent variable). Thus, if it is the covariates alone that cause more innovation during the interim, it would be reflected in the prediction and prediction interval. In fact, the covariates result in a smaller prediction than before. The resulting $p$-values for 26 services are even lower than for the previous predictions: further evidence that innovation changed during the interim.

One question remains: how sensitive are the predictions to the chosen parametric forms for the hazard? Here I re-do the prediction exercise using coefficient estimates from a Cox (1975) semiparametric proportional hazards model, using the fixed effects regressors. The dependent variable in this estimation is the interarrival times of innovation events. Flexibly modeling the 
interarrival process leads to a count distribution that can exhibit under- or overdispersion of any form. ${ }^{30}$ The coefficients are reported in the final column of Table 4 (note that the intercept is absorbed into the baseline hazard), and are close to the Poisson model. The prediction, based on the Cox coefficient estimates and a nonparametric estimate of the baseline hazard, is 15.0 plans and amendments filed during the interim, a bit lower than the prediction from the Poisson fixed effects models. ${ }^{31}$ The prediction is lower because the nonparametric estimate of the baseline interarrival hazard exhibits negative duration dependence, as opposed to the constant hazard imposed by the Poisson assumption. The 26 services actually introduced by the BOCs still lie outside of the $95 \%$ prediction interval (final row of Table 6). ${ }^{32}$ The results from the Cox model corroborate the other predictions: there is a very small chance that innovation did not change during the interim.

\subsection{The Regulatory Delay Model}

\subsubsection{Data}

Turn now to the regulatory delay submodel. The data set also contains the time to approval or withdrawal for each CEI plan, amendment, or waiver request. Most observations end with approval by the FCC, a few with withdrawal by the carrier. ${ }^{33}$ The approval delays were sizable: of the 68 spells ending in final approval, ${ }^{34}$ the average was 204 days and the longest (AT\&T SPECS waiver) was 22 months. Of the 14 withdrawn plans and ongoing delays, the delay averages 241 days. The longest ongoing delays (as of December 1997) are two CEI plans (Fast Packet and Internet Access) Ameritech first submitted in March 1995. ${ }^{35}$ Summary statistics of the data are in Table 7.

Other variables collected for each observation attempt to capture heterogeneity of services. Such variables include firm and plan fixed effects, whether it is a "me-too" filing, ${ }^{36}$ and whether the plan was refiled. One expects that delays will be longer the more complex the issues the CEI plan raises. I proxy plan complexity by counting the number of pages in the $F C C$ Record reporting the approval, because the report includes a discussion of the issues. The number of competitors 
that are affected by the plan may also affect the time to approval; I count the number of interested parties submitting comments for the public record.

\subsubsection{Estimation and Specification Checking}

The simplest duration model, the exponential model ( $\sigma=1$ and $\tau_{2}=0$; results not reported), is rejected statistically in favor of the Weibull models. I therefore use Weibull models to interpret coefficients and calculate predicted delay. The results for the fixed effects Weibull model $\left(\tau_{2}=0\right)$ are in Table 8, column one. The parameter $\sigma$ is estimated to be 0.613 and differs significantly from unity, rejecting the exponential model. ${ }^{37}$ The data exhibit positive duration dependence, implying that the longer the delay lasts, the more likely it is to end the next day (conditional on lasting as long as it has). The positive duration dependence may have a structural origin. The FCC has a legal obligation to act on filings in a timely matter, and the pressure may mount to approve filings if they have been delayed a long time.

To interpret the coefficients, recall from (9) that positive coefficients increase mean delay. ${ }^{38}$ The estimates indicate that me-too status garners $41 \%$ shorter delays. Amendments are approved $9 \%$ quicker and waivers $16 \%$ slower than plans. Refiling a plan not surprisingly increases delay, by $48 \%$. The average predicted mean duration is 226 days.

The heterogeneity parameter $\tau_{2}$ is allowed to vary in the second column of Table 8 . The results and likelihood from the generalized Weibull model differ little from the standard Weibull model in column one.

I also consider whether there is congestion in the regulatory approval process for CEI filings. There is much variation in the number of plans awaiting regulatory approval over the observation period. There are as many as 36 services stacked up in the "regulatory inbox" (shortly after the remand period, when the services introduced during the interim were filed en masse); see Figure 3. Does approval slow down when the regulatory system is backed up? It is of interest to separate the impact of plans already sitting on the regulator's desk when a new plan is filed, versus the 
impact of plans filed afterward. ${ }^{39}$ Two variables that are valid regressors in the model (refer to the discussion of equation (10) concerning endogenous duration covariates) are the number of services (from any firm) awaiting approval at the start of the spell and the number of new arrivals (from any firm) during the length of the spell. ${ }^{40} \mathrm{I}$ check for congestion by adding these two variables to the hazard specification.

The results of the congestion estimation are in Table 8, column three. Both congestion coefficients are negative, a surprising result, although only the number of new filings during the spell is significant. Neither effect is large, however. The elasticity of regulatory delay with respect to new filings is -0.29 , implying that an extra filing above average decreases the mean approval duration by less than a week. If these results are not spurious, ${ }^{41}$ an interpretation may be as follows. When services are stacked up in the regulatory inbox, the FCC relaxes its scrutiny of new plans a bit and approves them marginally quicker. Holding that effect constant, any filings arriving afterward speed up approval of a plan already in the inbox, perhaps because regulatory resources need to be shifted to the new arrivals.

Turning finally to the other covariates (the covariates estimation in Table 8), ${ }^{42}$ FCC Record pages appears to be a weak proxy for plan complexity. The coefficient is positive but not significant. The elasticity of 0.12 indicates that an extra page in the Record above the average of 6.4 increases mean delay by 4 days. The number of commenters is marginally significant; the elasticity of 0.046 indicates that an extra reply commenter above the average of 1.0 increases mean delay by only 1.2 days, indicating that opponents have limited power to slow down their rival's plan approvals. The predicted mean duration from this estimation is 214 days.

\subsection{The Jointly Correlated Model}

Turn now to the full model with unrestricted correlation allowed between the count and duration models. The results from the unrestricted, joint fixed effects and congestion estimations are in Table 9. In these estimations, unlike those reported in Tables 4 and $8, \rho$ is allowed to vary. In 
general, the coefficients are close to those from the independent estimations.

In the fixed effects estimation the correlation between the count and duration models is negative, as measured by $\rho$. Such negative correlation means that a shock leading to more innovation, and therefore more tariffs submitted to the regulator, has the effect of reducing regulatory delay time. This indirect congestion effect is similar to the direct congestion effects in the independent regulatory delay congestion estimation, where we included explicit covariates that elicited this effect. When these explicit covariates are omitted, as in the joint fixed effects estimation, then the direct effect shows up as negative unexplained correlation. Once the congestion variables are explicitly included in the joint estimation (the second column of Table 9), the residual correlation $\rho$ turns positive. Thus, net of the direct congestion effects in the model, there may be unobserved factors that cause both increased innovation and longer delays.

Although $\rho$ is significant in both joint estimations, LR tests of each unrestricted estimation against its restricted counterpart fail to reject the restricted models. Given this, and given the added complexity that correlation adds to simulating the joint model, it appears best to rely on the independent estimations for the comparison exercise in the next section.

\section{The Costs of the CEI Regime}

The predictions in section 5.1 have already provided evidence that innovation during the interim was $60-99 \%$ higher than would have been the case had regulation still been in place. In this section I include the impact of regulatory delay and ask a different question. Now that I have estimated the parameters for the entire model, I can compare the overall effect on service introductions (not just innovation) in the two periods. In Scenario 1, the interim period of lighter regulation never happens, and the CEI regime is in place for the entire observation period (1987-1997). In Scenario 2, the CEI regime never happens, and the interim conditions are in effect for the entire observation period. How many new services would one expect to have been introduced in each 
scenario? Comparing the two answers will measure the total effect that the regulatory regime had on innovation.

Scenario 1 employs the generalized Poisson fixed effects model for the arrival process (column two of Table 4) and the Weibull fixed effects model for the delay process (column one of Table 8). For Scenario 2, the projection is calculated by MLE of the simple Poisson fixed effects model using the interim data. This model is the only one identified with the interim data (see note 5). The Scenario 2 mean is 12.75 services created per year. Regulatory delay is zero for scenario 2 . Table 10 shows that in Scenario 1, the BOCs introduce a projected 92 services. At the end of the period, 5.7 services are still awaiting regulatory approval. ${ }^{43}$ In Scenario 2, the BOCs introduce a projected 140 services. Thus in Scenario 2, the firms create $52 \%$ more services and are able to introduce $62 \%$ more services than in Scenario 1. The average is 53.6 more services approved without the CEI regulation, and the standard deviation is small enough to assure that the difference is positive with high probability. ${ }^{44}$

In the steady state, the relevant comparison is between the innovation rates. As the observation period in the projection exercise grows, the difference between innovation and introduction goes away (in percentage terms). This is because (absent changes in covariates) the number of services created is proportional to the length of the observation period, while the absolute difference between innovation and introduction (services not approved in Table 10) is instead constant. In the context of the queuing model, in the steady state the output rate of the regulatory approval process equals the innovation rate. ${ }^{45}$ Nevertheless looking at introductions is interesting for two reasons. First, in any finite amount of time there is a wedge between innovation and introduction caused by regulatory delay. That wedge represents services that consumers could be enjoying but are notan opportunity cost of regulation. For example, AT\&T proposed to offer voice mail in the 1970's but was prevented by the FCC until after divestiture in 1984. Second, even in the steady state, if regulatory delay suddenly dropped to zero consumers would see a many more new services offered to them as the regulatory queue empties out. 
Exercises comparing counterfactual scenarios are only as good as the assumptions upon which they rest. Given the difficulties with such exercises, it is perhaps best to view this exercise as illustrative rather than to place stress on the numerical results per se. The results from the exercise illustrate the two ways that relaxed regulation benefits consumers of telecommunications services. First, many more new telecommunications information services were introduced when the CEI regime was suspended. The evidence is strong that the onerous requirements of the CEI regime prevented many services from being introduced. Second, suspending the CEI regime removed the long approval delays for CEI plans, meaning that each new service became available to consumers much sooner. Adding the CEI delay to the wait associated with tariffing any underlying basic services pushes the time to introduction out even farther. The elimination of these long introduction delays no doubt contributed to the BOCs' decisions to introduce more services (as the evidence from the innovation congestion estimation indicates).

\section{Discussion and Conclusions}

One would like to move beyond the mere counts and measure these increases in consumer welfare. This is not possible with the present data, any more than it is with most of the literature on patent counts. However, it is possible to do a "back of the envelope" calculation for voice messaging, one of the CEI services introduced by all the BOCs. One recent study (Hausman, 1997) estimates the consumer welfare gained from the introduction of voice mail by local telephone companies, finding that the structural prohibition against enhanced services cost consumers about $\$ 1.2$ billion per

year for voice messaging. Hausman's methodology is not undisputed $^{46}$ and I cannot extrapolate from this figure to other services (voice mail is one of the most successful CEI services). However, if the figure is valid, then at my model's predicted regulatory approval delay of 213 to 276 days (depending on which model in Table 8 is used) the CEI regime would have cost consumers an extra $\$ 690$ million to $\$ 910$ million in lost welfare for voice mail alone. 
Even if the BOCs would have introduced $62 \%$ more services if the regime had not been in place, I do not claim that welfare would have risen proportionally. Economic reasoning suggests that the services introduced during the interim were probably not of comparable importance to the services introduced under the CEI regime. The incremental services introduced during the interim - those that would not have been introduced under the CEI regime - most likely created less revenue for the BOCs since they were formerly deemed unprofitable to introduce. Accordingly, they were probably worth less to consumers as well. There is no way to estimate this difference in worth with the present data. I can, however, look at the types of plans that were filed for services introduced during the interim and compare them with those of the CEI regime. Of the 26 plans filed for the interim period, 12 of them $(46 \%)$ were amendments of previously approved plans. Amendments typically add new features to an existing service, and may be less beneficial to consumers than entirely new services. During the CEI regime, only $20 \%$ of filings are amendments. By this metric, it appears that the BOCs may have introduced less-important services.

The analysis implicitly assumes that the services introduced are truly new; the welfare impact of the regulation is blunted if they are not. For some of these services, consumers face substitutes offered by competitors. For example, Internet services offered by BOCs are included in these data, and consumers have many choices of Internet service providers. The case of Internet services is the exception rather than the rule, however. In general, the CEI services introduced by BOCs are not identical to any previously offered by competitors. Because the CEI services are seamlessly integrated with the BOCs networks, and therefore the subscriber's local telephone service, these services can offer features that competitors previously could not. It is very likely that even if some competitor already offered a related service, the BOC's offering was highly differentiated. In fact, one of the reasons the FCC instituted the CEI regime was because these services are unique, and because competitors feared the BOCs would therefore have an unfair advantage.

A final question concerns the nature of the experiment.

The estimation would stumble into the sequencing pitfall (identified by Sappington and Weis- 
man (1996)) if firms' actions are spurred by anticipated changes in the regulatory regime. This pitfall might apply to these data if, right before the ONA plans were approved, the BOCs held off on introducing new services in anticipation of the upcoming reduced cost of so doing. On the other end of the interim, the sequencing effect would also imply that fewer services would be introduced shortly after the regime was reinstated, if firms hurried services into the marketplace just before the regime change. Three facts provide evidence against this pitfall. First, some BOCs submitted CEI plans as shortly as a few weeks before and after the interim. Second, although there are only three new services during the period from Computer III reinstatement to the ONA plan approvals (see Figure 1), that number is well within the normal variation of the estimated innovation process. ${ }^{47}$ Third, a dummy variable for the periods just before and after the interim in the innovation regression is not significant.

Against the demonstrated costs to consumers of the CEI regime, what was there to balance? If regulators rationally and disinterestedly maximize social welfare, then they must have believed that the benefits of the CEI regime to consumers were large indeed. However, it appears that at the time the CEI regulation was initiated, the FCC did not consider the opportunity cost of the regulation: they compared the CEI regime to the previous structural separation regime (in which no enhanced services were allowed at all) instead of to a regime with even less regulation. I emphasize that this study is not a cost-benefits analysis; without more data on competitors the benefits are impossible to evaluate. However, the benefits of the regime appear to be slight: few requests for interconnection were ever made under the CEI regime, ${ }^{48}$ and competitors have shown little interest in the CEI plans in recent years. ${ }^{49}$ The FCC intended the CEI regime to be a short-term solution until the stricter ONA regime was fully in place. It took twelve years for the burdens of the CEI regime to be significantly reduced - a caution to those who would set up "temporary" regulation.

Of the three innovation-discouraging effects mentioned in the introduction, the estimations are able to address only the expected regulatory delay effect. Recall from section 5.1.3 that the estimated impact of reducing expected regulatory delay to nil is 3 to 6 new services created per 
year. Given that the projection exercise in section 6 estimated the innovation difference between the regulated and unregulated scenarios to be about 4.3 services per year, it appears that the expected delay effect accounts for much of the reduction in innovation under the CEI regime. One interesting extension to the present study would be to attempt to further disentangle these effects. As of February 1999, the BOCs are relieved from pre-filing CEI plans, but are still required to make the plans public. Therefore the effect of information revelation still exists under the present rules, while the effect from the direct costs of preparing the plan is reduced ${ }^{50}$ and the effect from approval delay is eliminated. It will be interesting to see if the rule change spurs the creation of new services. 


\section{Appendices}

\section{A CEI Services}

This section contains the CEI plans, amended plans, and waiver requests used in the statistical work.

Table 1: CEI Plans, Amendments, and Waivers

\begin{tabular}{|c|c|c|c|c|}
\hline CEI Filing & $\begin{array}{l}\text { Date } \\
\text { Filed }\end{array}$ & $\begin{array}{c}\text { Date } \\
\text { Approved } \\
\text { or W/drawn }\end{array}$ & $\begin{array}{l}\text { Included } \\
\text { in Arrival } \\
\text { Estimation }\end{array}$ & $\begin{array}{l}\text { Included } \\
\text { in Delay } \\
\text { Estimation }\end{array}$ \\
\hline \multicolumn{5}{|l|}{ Ameritech } \\
\hline Interactive Audiotex Svcs & $3 / 23 / 89$ & $9 / 21 / 89$ & Yes & Yes \\
\hline Enh Protocol Processing Svcs & $11 / 6 / 89$ & $5 / 24 / 90$ & Yes & Yes \\
\hline Alarm Monitoring & $3 / 13 / 95$ & $10 / 31 / 95$ & Yes $^{1}$ & $\mathrm{No}^{2}$ \\
\hline Fast Packet Data Svcs & $3 / 13 / 95$ & ongoing $^{3}$ & Yes $^{1}$ & $\mathrm{No}^{2}$ \\
\hline Fax Store \& Forward & $3 / 13 / 95$ & $10 / 31 / 95$ & Yes $^{1}$ & $\mathrm{No}^{2}$ \\
\hline Interactive Voice Response Svcs & $3 / 13 / 95$ & $10 / 31 / 95$ & Yes $^{1}$ & $\mathrm{No}^{2}$ \\
\hline Internet Access Svcs & $3 / 13 / 95$ & ongoing $^{3}$ & Yes $^{1}$ & $\mathrm{No}^{2}$ \\
\hline Voice Mail Messaging & $3 / 13 / 95$ & $10 / 31 / 95$ & Yes $^{1}$ & $\mathrm{No}^{2}$ \\
\hline Message Delivery Svc & $6 / 11 / 95$ & $12 / 15 / 95$ & Yes & Yes \\
\hline Personal Access Svc (PAS) & $9 / 1 / 95$ & $6 / 4 / 97$ & Yes & Yes \\
\hline $\begin{array}{l}\text { Voice Mail Messaging (minor } \\
\text { amendment) }\end{array}$ & $8 / 22 / 96$ & $10 / 28 / 96$ & Yes & Yes \\
\hline Fax Store \& Forward (amended) & $9 / 27 / 96$ & $12 / 4 / 96$ & Yes & Yes \\
\hline Reverse Search (waiver) & $10 / 25 / 96$ & $3 / 24 / 97$ & Yes & Yes \\
\hline $\begin{array}{l}\text { Voice Mail Messaging (supplement to } \\
\text { minor amendment) }\end{array}$ & $10 / 28 / 96$ & $11 / 14 / 96$ & Yes & Yes \\
\hline Payphone Svc & $11 / 27 / 96$ & $4 / 15 / 97$ & $\mathrm{No}^{4}$ & Yes \\
\hline Electronic Vaulting Svc & $2 / 27 / 97$ & $12 / 31 / 97$ & Yes & Yes \\
\hline \multicolumn{5}{|l|}{ Bell Atlantic } \\
\hline Message Storage & $3 / 6 / 87$ & $2 / 18 / 88$ & Yes & Yes \\
\hline Message Storage (amended) & $3 / 21 / 88$ & $5 / 23 / 88$ & Yes & Yes \\
\hline Coin Messaging (waiver) & $4 / 1 / 88$ & $9 / 29 / 88$ & Yes & Yes \\
\hline Intellgate/Videotex Gateway & $3 / 30 / 88$ & $9 / 30 / 88$ & Yes & Yes \\
\hline Voice Messaging Svc & $3 / 30 / 88$ & $9 / 30 / 88$ & Yes & Yes \\
\hline $\begin{array}{l}\text { Intellgate/Videotex Gateway } \\
\text { (amended) }\end{array}$ & $10 / 11 / 88$ & $1 / 30 / 89$ & Yes & Yes \\
\hline
\end{tabular}

\footnotetext{
${ }^{1}$ Plan filed after the remand to indicate a service had been introduced during the interim. Used only for calculation of mean arrival rate for scenario 2 in Section 6 .

${ }^{2}$ Plans filed en masse after the interim are excluded from the delay analysis because the FCC approved them all at the same time, indicating an exception to the usual approval process.

${ }^{3}$ As of end of 1997.

${ }^{4}$ Filing required by the FCC - does not reflect a new service.
} 
Table 1: CEI Plans, Amendments, and Waivers

\begin{tabular}{|c|c|c|c|c|}
\hline CEI Filing & $\begin{array}{l}\text { Date } \\
\text { Filed } \\
\end{array}$ & $\begin{array}{c}\text { Date } \\
\text { Approved } \\
\text { or } \mathrm{W} / \text { drawn } \\
\end{array}$ & $\begin{array}{c}\text { Included } \\
\text { in Arrival } \\
\text { Estimation }\end{array}$ & $\begin{array}{c}\text { Included } \\
\text { in Delay } \\
\text { Estimation }\end{array}$ \\
\hline Protocol Processing Svcs & 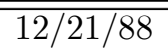 & $3 / 31 / 89$ & 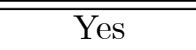 & 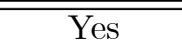 \\
\hline Electronic Data Interchange Svcs & $1 / 30 / 89$ & $6 / 9 / 89$ & Yes & Yes \\
\hline Data Processings \& Storage Svcs & $6 / 6 / 89$ & $12 / 13 / 89$ & Yes & Yes \\
\hline Radio-Based Enhanced Svcs & $3 / 22 / 90$ & $7 / 27 / 90$ & Yes & Yes \\
\hline Coin Messaging Delivery Svc (waiver) & $2 / 12 / 91$ & $6 / 4 / 91$ & Yes & Yes \\
\hline Enhanced Video Svcs & $1 / 27 / 95$ & $6 / 9 / 95$ & Yes & Yes \\
\hline Protocol Processing Svcs (amendment) & $3 / 13 / 95$ & $10 / 31 / 95$ & Yes $^{1}$ & $\mathrm{No}^{2}$ \\
\hline Internet Access Svc & $3 / 8 / 96$ & $6 / 6 / 96$ & Yes & Yes \\
\hline Payphone Svc & $1 / 6 / 97$ & $4 / 15 / 97$ & $\mathrm{No}^{4}$ & Yes \\
\hline Internet Access Svc (amendment) & $5 / 5 / 97$ & ongoing 3 & Yes & Yes \\
\hline Intranet Management Svc & $10 / 3 / 97$ & ongoing $^{3}$ & Yes & Yes \\
\hline \multicolumn{5}{|l|}{ BellSouth } \\
\hline Voice Messaging Svc & $3 / 18 / 88$ & $8 / 31 / 88$ & Yes & Yes \\
\hline VMS (amended) & $8 / 31 / 88$ & $12 / 23 / 88$ & Yes & Yes \\
\hline Coin Messaging (waiver) & $10 / 7 / 88$ & $2 / 15 / 89$ & Yes & Yes \\
\hline Synchronous Protocol Processing Svcs & $8 / 19 / 88$ & $2 / 15 / 89$ & Yes & Yes \\
\hline Gateway Svcs (amended) & $11 / 2 / 88$ & $3 / 30 / 89$ & Yes & Yes \\
\hline Gateway Svcs (further amendment) & $3 / 30 / 89$ & $5 / 25 / 89$ & Yes & Yes \\
\hline $\begin{array}{l}\text { Synchronous Protocol Processing Svcs } \\
\text { (amendment) }\end{array}$ & $5 / 24 / 89$ & $9 / 14 / 89$ & Yes & Yes \\
\hline Account Code Billing (waiver) & $3 / 7 / 91$ & $5 / 27 / 92$ & Yes & Yes \\
\hline Voice Messaging Svcs (amended) & $6 / 1 / 93$ & $7 / 14 / 93$ & Yes & Yes \\
\hline Gateway Svcs (amended) & $3 / 13 / 95$ & $10 / 31 / 95$ & Yes $^{1}$ & $\mathrm{No}^{2}$ \\
\hline $\begin{array}{l}\text { Synchronous Protocol Processing Svcs } \\
\text { (amendment) }\end{array}$ & $3 / 13 / 95$ & $10 / 31 / 95$ & Yes $^{1}$ & $\mathrm{No}^{2}$ \\
\hline Reverse Search (waiver) & $3 / 1 / 96$ & $7 / 3 / 96$ & Yes & Yes \\
\hline Payphone Svc & $11 / 22 / 96$ & $4 / 15 / 97$ & $\mathrm{No}^{4}$ & Yes \\
\hline $\begin{array}{l}\text { Gateway Svcs and Synchronous Proto- } \\
\text { col Processing Svcs (amended) \& wvr }\end{array}$ & $8 / 26 / 97$ & ongoing ${ }^{3}$ & Yes & Yes \\
\hline \multicolumn{5}{|l|}{ NYNEX } \\
\hline Info-Look/Videotex Gateway (waiver) & $5 / 11 / 88$ & $10 / 5 / 88$ & Yes & Yes \\
\hline Voice Messaging Svc & $6 / 21 / 88$ & $1 / 12 / 89$ & Yes & Yes \\
\hline Protocol Processing Svcs & $5 / 17 / 89$ & $1 / 4 / 90$ & Yes & Yes \\
\hline Electronic Info Svcs. & $3 / 13 / 95$ & $10 / 31 / 95$ & Yes $^{1}$ & $\mathrm{No}^{2}$ \\
\hline Voice Messaging Svcs (amended) & $3 / 13 / 95$ & $10 / 31 / 95$ & Yes $^{1}$ & $\mathrm{No}^{2}$ \\
\hline Audiotext Info Srvcs & $7 / 28 / 95$ & $1 / 23 / 96$ & Yes & Yes \\
\hline Custom Announcement Svcs & $7 / 28 / 95$ & $1 / 23 / 96$ & Yes & Yes \\
\hline Remote Data Processing Svcs & $7 / 28 / 95$ & $1 / 23 / 96$ & Yes & Yes \\
\hline Payphone Svc & $1 / 3 / 97$ & $4 / 15 / 97$ & $\mathrm{No}^{4}$ & Yes \\
\hline
\end{tabular}

\footnotetext{
${ }^{5}$ These were filed as two CEI plans; they are counted as one because the plans were filed to indicate that technologies converged.
} 
Table 1: CEI Plans, Amendments, and Waivers

\begin{tabular}{|c|c|c|c|c|}
\hline CEI Filing & $\begin{array}{l}\text { Date } \\
\text { Filed }\end{array}$ & $\begin{array}{c}\text { Date } \\
\text { Approved } \\
\text { or } \mathrm{W} / \text { drawn }\end{array}$ & $\begin{array}{c}\text { Included } \\
\text { in Arrival } \\
\text { Estimation } \\
\end{array}$ & $\begin{array}{c}\text { Included } \\
\text { in Delay } \\
\text { Estimation }\end{array}$ \\
\hline Voice Mail Svc & $7 / 2 / 87$ & $2 / 18 / 88$ & Yes & Yes \\
\hline Voice Mail Svc (amended) & $3 / 21 / 88$ & $5 / 23 / 88$ & Yes & Yes \\
\hline Electronic Messaging Svcs & $6 / 20 / 88$ & $2 / 21 / 89$ & Yes & Yes \\
\hline Videotex Gateway svc & $9 / 23 / 88$ & $4 / 7 / 89$ & Yes & Yes \\
\hline Voice Store \& Forward Svc & $11 / 18 / 88$ & $5 / 22 / 89$ & Yes & Yes \\
\hline Protocol Conversion Sves & $3 / 19 / 90$ & $10 / 25 / 90$ & Yes & Yes \\
\hline Protocol Conversion Svcs (amended) & $3 / 19 / 92$ & $5 / 21 / 93$ & Yes & Yes \\
\hline Voice Messaging Svcs (amended) & $5 / 1 / 92$ & $5 / 29 / 92$ & Yes & Yes \\
\hline Electronic Messaging Svcs (amended) & $3 / 13 / 95$ & $10 / 31 / 95$ & $\mathrm{Yes}^{1}$ & $\mathrm{No}^{2}$ \\
\hline $\begin{array}{l}\text { Enhanced Protocol, Code, and Format } \\
\text { Conv. Svc }\end{array}$ & $3 / 13 / 95$ & $10 / 31 / 95$ & Yes $^{1}$ & $\mathrm{No}^{2}$ \\
\hline Fax Store and Forward & $3 / 13 / 95$ & $10 / 31 / 95$ & Yes $^{1}$ & $\mathrm{No}^{2}$ \\
\hline VMS (amended) & $3 / 13 / 95$ & $10 / 31 / 95$ & Yes $^{1}$ & $\mathrm{No}^{2}$ \\
\hline Voice Store \& Forward (amended) & $3 / 13 / 95$ & $10 / 31 / 95$ & Yes $^{1}$ & $\mathrm{No}^{2}$ \\
\hline Payphone Svc & $12 / 26 / 96$ & $4 / 15 / 97$ & $\mathrm{No}^{4}$ & Yes \\
\hline \multicolumn{5}{|l|}{ SWBT } \\
\hline Voice Messaging Svcs & $4 / 1 / 88$ & $9 / 29 / 88$ & Yes & Yes \\
\hline Gateway (waiver) & $8 / 24 / 88$ & $1 / 30 / 89$ & Yes & Yes \\
\hline Protocol Conversion Svcs & $12 / 31 / 88$ & $3 / 9 / 89$ & Yes & Yes \\
\hline Voice Messaging Svcs (amended) & $4 / 17 / 90$ & $7 / 23 / 90$ & Yes & Yes \\
\hline Payment Processing Svcs & $3 / 13 / 95$ & $10 / 31 / 95$ & Yes $^{1}$ & $\mathrm{No}^{2}$ \\
\hline Protocol Conversion Svcs (Amendment) & $3 / 13 / 95$ & $10 / 31 / 95$ & Yes $^{1}$ & $\mathrm{No}^{2}$ \\
\hline Voice Messaging Svcs (amended) & $3 / 13 / 95$ & $10 / 31 / 95$ & Yes $^{1}$ & $\mathrm{No}^{2}$ \\
\hline Fax Svc & $8 / 3 / 95$ & $5 / 7 / 96$ & Yes & Yes \\
\hline Internet Access Svc & $8 / 3 / 95$ & $1 / 11 / 96$ & Yes & Yes \\
\hline Fax Svc (minor amendment) & $5 / 7 / 96$ & $6 / 11 / 96$ & Yes & Yes \\
\hline PC Backup \& Recovery & $8 / 3 / 95$ & $6 / 11 / 96$ & Yes & Yes \\
\hline Reverse Search (waiver) & $1 / 16 / 96$ & $7 / 1 / 96$ & Yes & Yes \\
\hline Basic Payphone & $12 / 30 / 96$ & $4 / 15 / 97$ & $\mathrm{No}^{4}$ & Yes \\
\hline Interactive Call Manager & $8 / 15 / 96$ & $5 / 8 / 97$ & Yes & Yes \\
\hline Security Svc & $4 / 4 / 96$ & $5 / 16 / 97$ & Yes & Yes \\
\hline Internet Support Svcs & $6 / 21 / 96$ & ongoing 3 & Yes & Yes \\
\hline Payphone (minor amendment) & $7 / 11 / 97$ & ongoing $^{3}$ & Yes & Yes \\
\hline \multicolumn{5}{|l|}{ U S West } \\
\hline Voice Messaging Svcs & $5 / 13 / 88$ & $1 / 13 / 89$ & Yes & Yes \\
\hline Protocol Processing Svcs & $2 / 24 / 89$ & $7 / 13 / 89$ & Yes & Yes \\
\hline Voice Store \& Forward & $10 / 24 / 89$ & $6 / 6 / 90$ & Yes & Yes \\
\hline Fax Store \& Forward & $12 / 15 / 89$ & $6 / 6 / 90$ & Yes & Yes \\
\hline Audiotex & $3 / 13 / 95$ & $10 / 31 / 95$ & Yes $^{1}$ & $\mathrm{No}^{2}$ \\
\hline Enhanced Fax Svcs & $3 / 13 / 95$ & $10 / 31 / 95$ & Yes $^{1}$ & $\mathrm{No}^{2}$ \\
\hline Electronic Messaging Svcs & $3 / 13 / 95$ & $10 / 31 / 95$ & $\mathrm{Yes}^{1}$ & $\mathrm{No}^{2}$ \\
\hline On-Line DB access & $3 / 13 / 95$ & $10 / 31 / 95$ & Yes $^{1}$ & $\mathrm{No}^{2}$ \\
\hline Protocol Processing Svcs (amended) & $3 / 13 / 95$ & $10 / 31 / 95$ & Yes $^{1}$ & $\mathrm{No}^{2}$ \\
\hline
\end{tabular}


Table 1: CEI Plans, Amendments, and Waivers

\begin{tabular}{|c|c|c|c|c|}
\hline CEI Filing & $\begin{array}{l}\text { Date } \\
\text { Filed }\end{array}$ & $\begin{array}{c}\text { Date } \\
\text { Approved } \\
\text { or } \mathrm{W} / \text { drawn }\end{array}$ & $\begin{array}{l}\text { Included } \\
\text { in Arrival } \\
\text { Estimation }\end{array}$ & $\begin{array}{c}\text { Included } \\
\text { in Delay } \\
\text { Estimation }\end{array}$ \\
\hline Voice Messaging Svcs (amended) & $3 / 13 / 95$ & $\overline{~ 10 / 31 / 95}$ & $\overline{\text { Yes }^{1}}$ & $\overline{\mathrm{No}^{2}}$ \\
\hline Reverse Search (waiver) & $4 / 4 / 94$ & $11 / 6 / 95$ & Yes & Yes \\
\hline Voice Messaging Svcs (amended) & $9 / 13 / 96$ & ongoing $^{3}$ & Yes & Yes \\
\hline Payphone & $1 / 6 / 97$ & $4 / 15 / 97$ & $\mathrm{No}^{4}$ & Yes \\
\hline \multicolumn{5}{|l|}{$\mathbf{A T} \& \mathbf{T}^{6}$} \\
\hline Subaccount Billing Sves (waiver) & $6 / 10 / 87$ & $8 / 19 / 87$ & Yes & Yes \\
\hline Transaction Svcs & $10 / 26 / 87$ & $5 / 11 / 88$ & Yes & Yes \\
\hline CODEC conversion & $12 / 18 / 87$ & $7 / 29 / 88$ & Yes & Yes \\
\hline FTS 2000 & $1 / 24 / 89$ & $6 / 15 / 89$ & Yes & Yes \\
\hline Enhanced Svcs complex & $3 / 30 / 89$ & $9 / 13 / 89$ & Yes & Yes \\
\hline $\begin{array}{l}\text { DIAL IT } 900 \text { Svc Call Count Arrange- } \\
\text { ments (waiver) }\end{array}$ & $8 / 18 / 89$ & $10 / 6 / 89$ & Yes & Yes \\
\hline Enhanced Packet Svcs & $6 / 23 / 89$ & $2 / 2 / 90$ & Yes & Yes \\
\hline Transaction Svcs (amended) & $6 / 7 / 90$ & $12 / 18 / 90$ & Yes & Yes \\
\hline ESC (amended) & $2 / 13 / 91$ & $8 / 8 / 91$ & Yes & Yes \\
\hline SPECS Enh. Svcs (waiver) & $10 / 31 / 91$ & $8 / 27 / 93$ & Yes & Yes \\
\hline
\end{tabular}

\section{B Details of the Projection Exercise in Section 6}

This supplement contains further details on the calculations underlying the predictions in The Costs of the CEI Regime section, and will be made available upon request to interested readers (as indicated in a footnote in the paper). The innovation (count) model and the regulatory delay (selection and duration) models compose a $G / G / \infty$ queuing system ${ }^{7}$ (Prieger (1999) explicates this equivalence in detail). To know how many services are introduced to consumers, one needs to calculate what the output of the queuing system is given the estimated parameters. When innovation (arrivals, in the parlance of queuing theory) follows a homogeneous Poisson process, an existing result from queuing theory provides this answer. I first present this result, and then show

\footnotetext{
${ }^{6}$ After 1991, the FCC treated enhanced services from AT\&T differently than those from the BOCs. To preserve comparability, new servies by AT\&T after 1991 are not included in the data set.

${ }^{7} G$ is a catch-all symbol for a general distribution. The notation means the arrivals and processing time follow an unspecified distribution, and that there are an infinite number of processors (i.e., in this model the services awaiting regulatory approval do not queue up).
} 
how it must be modified for the present model, in which innovation is inhomogeneous and contains random effects. All results in this section are for the independent model $(\rho=0)$.

To find the output process of a homogeneous Poisson arrival $(M / G / \infty ; M$ for Markovian $)$ queue, let $\lambda$ be the rate parameter for the Poisson arrival stochastic process and let $G$ be the CDF of the delay time process. Then the output process of a $M / G / \infty$ queue is a non-homogeneous Poisson stochastic process with intensity $\kappa(t)=\lambda G(t)$ (Ross, 1983, p.39). The number of events occurring from this output process in $[0, \tau]$ is a Poisson random variable with mean

$$
m(\tau)=\int_{0}^{\tau} \kappa(s) d s
$$

(Do not confuse this $\tau$ with $\tau_{1}$ and $\tau_{2}$ from the innovation and regulatory delay model in section 4 of the paper.)

Recall that in my model innovation is inhomogenous: the rate $\lambda_{t k \ell}=e^{u_{1 t}} \exp \left(\alpha_{1 k}+\alpha_{2 \ell}+\mathbf{x}_{t}^{\prime} \boldsymbol{\beta}\right)$ changes each period due to the covariates and the random effect $u_{1 t}$. The number of arrivals in a year (conditional on $u_{1 t}$ ) follow a Poisson distribution with mean $\lambda_{t k \ell}$. The inhomogeneity of innovation means that (13) must be modified for the model in the paper. First, note that conditional on $\left\{u_{1 t}\right\}_{t=1}^{T}$, each period is, in isolation, a simple $M / G / \infty$ queue (i.e., conditional on $u_{1 t}$ the arrival process is Poisson and the arrival rate is constant within the period). Let $\tau$ be the time of the end of the observation period. Then the question is what is the contribution of the $M / G / \infty$ queue in period $t$ to the number of exits from the system at time $\tau$ ? Once this answer is known (conditional on $u_{1 t}$ ), the total number of exits at time $\tau$ will be found as the sum of the contributions from each previous period. To find an answer unconditional on $\left\{u_{1 t}\right\}$ will then require integrating out $\left\{u_{1 t}\right\}$.

To find the contribution of period $t$ to the number of exits from the system at time $\tau$, define $s_{t}$ to be the length of period $t$ and $\varsigma_{t}$ to be the length of time between the end of period $t$ and $\tau$. During period $t$, some arrivals during that period will exit during that period, and some will exit at a later time. Denote the number of arrivals during period $t$ as $n_{t}$, the number of current-period 
arrivals that exit during the period as $k_{t}$, the number of current-period arrivals left in the system at the end of the period as $m_{t} \equiv n_{t}-k_{t}$, and the number of $m_{t}$ that exit before time $\tau$ as $l_{t}$ (drop the $k \ell$ subscripts for now, for notational simplicity). We are interested in the mean and variance of $k_{t}+l_{t}$, which can be found by first conditioning on $\left\{u_{1 t}\right\}$ and then unconditionally. At this point we need some more notation. Let $Q_{t}\left(u_{1 t}\right) \equiv k_{t}+l_{t} \mid u_{1 t}, Q_{t}^{m}\left(u_{1 t}\right) \equiv E_{k_{t}, l_{t}}\left(Q_{t}\left(u_{1 t}\right)\right)$, and $Q_{t}^{v}\left(u_{1 t}\right)=\operatorname{Var}_{k_{t}, l_{t}}\left(Q_{t}\left(u_{1 t}\right)\right)$.

From (13) it follows that

$$
k_{t} \mid u_{1 t} \sim \operatorname{Poisson}\left(\text { mean }=\lambda_{t} \int_{0}^{s_{t}} G(s) d s\right)
$$

where $G$ is the cdf of the duration process that describes regulatory delay (recall that $u_{1 t}$ is embedded in $\lambda_{t}$ ). Note that $G$ is the same for all $t$.

Now find the distribution of $l_{t} \mid u_{1 t}$. Given $m_{t}$ and $u_{1 t}$, the probability that $l_{t}=l$ at time $\tau$ is

$$
\left.P_{l}\right|_{m_{t}}\left(\varsigma_{t}\right)=\left(\begin{array}{c}
m_{t} \\
m_{t}-l
\end{array}\right) G\left(\varsigma_{t}\right)^{l}\left[1-G\left(\varsigma_{t}\right)\right]^{m_{t}-l} .
$$

This expression follows from standard probability arguments (Ross, 1983, p.39): with probability $G\left(\varsigma_{t}\right)$ an arrival exits by time $\varsigma_{t}$ and with probability $1-G\left(\varsigma_{t}\right)$ an arrival does not exit by time $\varsigma_{t}$; these events are independent, and the binomial coefficient term reflects all the combinations of possible events. The unconditional probability that $l_{t}=l$ depends on the distribution of $m_{t}$. Define $\Lambda_{t} \equiv \lambda_{t}\left[s_{t}-\int_{0}^{s_{t}} G(s) d s\right]$; then $m_{t} \mid u_{1 t} \sim$ Poisson(mean= $\left.\Lambda_{t}\right)($ Ross, 1983, 38-39). Then the unconditional probability that $l_{t}=l$ unconditional on $m_{t}$ at time $\tau$ is

$$
\begin{aligned}
E_{m_{t}}\left(\left.P_{l}\right|_{m_{t}}\left(\varsigma_{t}\right)\right) & =\sum_{l=0}^{m_{t}}\left(\begin{array}{c}
m_{t} \\
m_{t}-l
\end{array}\right) G\left(\varsigma_{t}\right)^{l}\left[1-G\left(\varsigma_{t}\right)\right]^{m_{t}-l} \frac{e^{-\Lambda_{t}}}{m_{t} !} \Lambda_{t}^{m_{t}} \\
& =\exp \left(\Lambda_{t} G\left(\varsigma_{t}\right)\right)\left[\Lambda_{t} G\left(\varsigma_{t}\right)\right]^{l} / l !
\end{aligned}
$$

from which it is clear that

$$
l_{t} \mid u_{1 t} \sim \operatorname{Poisson}\left(\text { mean }=\Lambda_{t} G\left(\varsigma_{t}\right)\right)
$$

Putting the pieces together, from (14) and (18) we have

$$
k_{t}+l_{t} \mid u_{1 t} \sim \text { Poisson }\left(\text { mean }=\lambda_{t}\left(G\left(\varsigma_{t}\right) s_{t}+\left[1-G\left(\varsigma_{t}\right)\right] \int_{0}^{s_{t}} G(s) d s\right)\right)
$$


so that

$$
Q_{t k \ell}^{m}\left(u_{1 t}\right)=\lambda_{t k \ell}\left(G_{k \ell}\left(\varsigma_{t}\right) s_{t}+\left[1-G_{k \ell}\left(\varsigma_{t}\right)\right] \int_{0}^{s_{t}} G_{k \ell}(s) d s\right)
$$

and

$$
Q_{t k \ell}^{v}\left(u_{1 t}\right)=Q_{t k \ell}^{m}\left(u_{1 t}\right)
$$

(recall that the Poisson variance equals the mean), where now the subscripts $k$ (for firms) and $\ell$ (for plan types) are reintroduced into the notation.

Let $Q=\sum_{t=1}^{T} \sum_{k \in K} \sum_{\ell \in L} k_{t k \ell}+l_{t k \ell}$ be the total number of exits from the system at time $\tau$. Define $Q^{m} \equiv E(Q)$ and $Q^{v}=\operatorname{Var}(Q)$. Since the $\left\{u_{1 t}\right\}$ are mutually independent and $E\left(\lambda_{t k \ell}\right)=$ $\lambda_{t k \ell}^{0}=\exp \left(\alpha_{1 k}+\alpha_{2 \ell}+\mathbf{x}_{t}^{\prime} \boldsymbol{\beta}\right), Q^{m}$ is found from (20) as

$$
Q^{m}=\sum_{t=1}^{T} \sum_{k \in K} \sum_{\ell \in L} \exp \left(\alpha_{1 k}+\alpha_{2 \ell}+\mathbf{x}_{t}^{\prime} \boldsymbol{\beta}\right)\left(G_{k \ell}\left(\varsigma_{t}\right) s_{t}+\left[1-G_{k \ell}\left(\varsigma_{t}\right)\right] \int_{0}^{s_{t}} G_{k \ell}(s) d s\right)
$$

Note that if there are no regulatory delays, so that $G=1$, then $Q_{m}=\sum_{t=1}^{T} \sum_{k \in K} \sum_{\ell \in L} \lambda_{t k \ell}^{0} s_{t}$, the simple number of arrivals over the observation period. The second term in parentheses in (22) is bounded between 0 and 1 , and is an adjustment factor to reflect that not all arrivals will have exited the system by the end of the observation period when there is regulatory delay.

Furthermore, independence of the $\left\{u_{1 t}\right\}$ and the formula for the relationship between marginal and conditional variance implies that

$$
Q^{v}=\sum_{t=1}^{T} \operatorname{Var}_{u_{1 t}}\left[Q_{t}^{m}\left(u_{1 t}\right)\right]+E_{u_{1 t}}\left[Q_{t}^{v}\left(u_{1 t}\right)\right]
$$

where $Q_{t}^{m}\left(u_{1 t}\right) \equiv \sum_{k \in K} \sum_{\ell \in L} Q_{t k \ell}^{m}\left(u_{1 t}\right)$ and $Q_{t}^{v}\left(u_{1 t}\right) \equiv \sum_{k \in K} \sum_{\ell \in L} Q_{t k \ell}^{v}\left(u_{1 t}\right)$. Making use of (20) and (21) yields

$$
Q^{v}=\left(e^{\tau_{1}^{2}}-1\right) \sum_{t=1}^{T}\left[\sum_{k \in K} \sum_{\ell \in L} \lambda_{t k \ell}^{0}\left(G_{k \ell}\left(\varsigma_{t}\right) s_{t}+\left[1-G_{k \ell}\left(\varsigma_{t}\right)\right] \int_{0}^{s_{t}} G_{k \ell}(s) d s\right)\right]^{2}+Q^{m}
$$

Since the $u_{1 t}$ are normally distributed, $\lambda_{t k \ell}$ is lognormally distributed. The $\left(e^{\tau_{1}^{2}}-1\right)$ part of $Q^{v}$ is the variance of the lognormal $\exp \left(u_{1 t}\right)$. 
Now all that remains is the find an expression for $G$. When $G$ is the cdf of the Weibull distribution (as in the fixed effects Weibull model in the first column of Table 8, used for Scenario 1), the integral appearing in (22) and (24) must be calculated numerically. Conditional on rate $\mu_{i}$, the Weibull cdf is given by equation (8) in the paper. This is the function denoted $G(\tau)$ above.

For the Scenario 1 prediction from The Costs of the CEI Regime section of the paper (the CEI regime for all of the observation period), I set $\tau=$ (11 years) and evaluate $Q^{m}$ from (22) with this value of $\tau$ and the estimates $(\hat{\boldsymbol{\alpha}}, \hat{\boldsymbol{\beta}}, \hat{\boldsymbol{\gamma}}, \hat{\sigma})$ from the Generalized Poisson fixed effects estimation in Table 4 of the paper. The calculation uses the BOCs only (not AT\&T), given that AT\&T's data are only partially observed: $K=\{2, \ldots, 8\}$. The remand period indicator variable, the only $x_{t}$ in this model, is set to 0 for all periods except those from 1995-1997. Evaluating the integrals in $Q^{m}$ required numerical integration in Fortran (note that there are 924 integrals to be calculated: 44 quarters $\times 7$ firms $\times 3$ plan types). For Scenario 2 (no CEI regulation during the observation period), the only model that is identified is a homogeneous Poisson fixed effects model, because the only data available from the interim are $n_{k \ell}$, the total counts from the interim for each firm and plan type. Formulae (22) and (24) also hold for this model if $\tau_{1}$ is set to zero and $G_{k \ell}=1$ (i.e., no regulatory delay).

The values of $Q^{m}$ for the two scenarios are reported in Table 9 as Projected Services Introduced. The standard deviation reported in the table is the square root of $Q^{v}$ from (24). Note that $Q^{v}$ accounts only for variation from $\left\{k_{t}, l_{t}, u_{1 t}\right\}$, not from estimation error in $\left(\hat{\boldsymbol{\alpha}}, \hat{\boldsymbol{\beta}}, \hat{\boldsymbol{\gamma}}, \hat{\sigma}, \hat{\tau}_{1}\right)$. Note further that since $Q$ is not Normally distributed, the usual confidence interval based on $Q^{v}$ will not have the usual coverage probability. However, the Poisson distribution is well-approximated by the Normal distribution when the mean is large. Therefore, if $Q^{m}$ is relatively large compared to the variance of $u_{1 t}, Q$ is approximately Poisson and approximately Normal, and $\left[Q^{m}-2 \sqrt{Q^{v}}, Q^{m}+\right.$ $2 \sqrt{Q^{v}}$ will have approximately $95 \%$ coverage probability.

The confidence interval for the Difference column in the same table is even more complicated. Even without the $u_{1 t}$, the Difference random variable is a difference of two Poisson random vari- 
ables, the distribution of which has no closed form expression (Johnson, Kotz and Kemp, 1993, pp.191-192). Again, however, if the mean difference is relatively large compared to the variance of $u_{1 t}$, the confidence interval based on the usual pivots will have approximately correct coverage probability.

\section{Calculating Expected Regulatory Delay}

The congestion estimations include a measure of expected regulatory delay. Here I explain the construction of this variable. A few features that should be part of such a variable are:

- Expected delay should be conditional on information available to the firms, which is constantly changing.

- Information on ongoing as well as completed delays should be incorporated into the expectation.

- Expected delay should be updated in some systematic fashion as new information arrives.

- More recently revealed information should be given more weight than old information, since regulatory behavior may evolve over time.

My expected regulatory delay variable has all these features. Begin by assuming that firms hold a common belief that regulatory delays are iid draws from a distribution $f_{Y}(y)$. Assume further that firms believe $f_{Y}$ is the exponential distribution with rate $\lambda$, so that a random sample of completed and ongoing delays $\mathbf{y}=y_{1}, \ldots, y_{N}$ has distribution

$$
f_{\mathbf{Y}}(\mathbf{y} \mid \lambda)=\prod_{i=1}^{N}\left[\lambda \exp \left(-\lambda y_{i}\right)\right]^{d_{i}}\left[\exp \left(-\lambda y_{i}\right)\right]^{1-d_{i}}
$$

where $d_{i}$ is an indicator that is 1 if the spell is completely observed and 0 if ongoing. Given $\lambda$, the expected mean delay for an uncensored spell is $1 / \lambda$. The firms all hold the common prior belief 
that the rate parameter $\lambda$ has prior distribution

$$
\pi(\lambda)=\mu^{2} \lambda \exp (-\lambda \mu)
$$

This prior is a Gamma distribution with shape parameter $\alpha=2$ (this distribution is also known as an Erlang distribution). I emphasize that these beliefs are simple for the purpose of tractability. This distribution (Gamma) is the conjugate prior for the exponential distribution, and so admits simple analytical results.

Based on the information revealed through $\mathbf{y}$, the firms update their belief about the distribution of $\lambda$, resulting in a posterior distribution for $\lambda$, denoted $\pi(\lambda \mid \mathbf{y})$, which is found from Bayes' rule as

$$
\pi(\lambda \mid \mathbf{y})=\frac{\pi(\lambda) f_{\mathbf{Y}}(\mathbf{y} \mid \lambda)}{\int \pi(\lambda) f_{\mathbf{Y}}(\mathbf{y} \mid \lambda) d \lambda}=\frac{\lambda^{N_{1}-1} \exp (-\lambda[\mu+\tilde{Y}])(\tilde{Y}+\mu)^{N_{1}+1}}{\Gamma\left(N_{1}+1\right)}
$$

also Gamma distributed, where $N_{1}$ is the number of completed durations $\left(N_{1}=\sum_{i=1}^{N} d_{i}\right)$ and $\tilde{Y}$ is the sum of the durations, completed or not $\left(\tilde{Y}=\sum_{i=1}^{N} y_{i}\right)$.

Given the posterior distribution for $\lambda,(27)$, the posterior expected delay is

$$
x_{e d}=\int_{0}^{\infty} \frac{1}{\lambda} \pi(\lambda \mid \mathbf{y}) d \lambda=\frac{\mu+\tilde{Y}}{N_{1}}
$$

Two questions remain: how do we choose $\mu$, and how can we incorporate the fourth bullet point above? First, on $\mu$, the parameter in the prior distribution (26). One can calculate the prior expected delay to be $\int_{0}^{\infty} \lambda^{-1} \pi(\lambda) d \lambda=\mu$. Thus $\mu$ is the initial belief about regulatory delay before any actual delays are revealed. In the spirit of rational expectations I assume that the firms have correct expectations at the beginning and set $\mu$ equal to the average delay that is seen over the first two years of data. The influence of the initial assumption dies out fairly quickly over time. Second, consider ways to weight more recent information more than old information. Note that expected regulatory delay $(28)$ is an average of $\mu$ and the revealed information $\tilde{Y}$, and that all observations composing $\tilde{Y}$ are weighted equally. I do the following instead. The value of $x_{e d}$ in a given period $t$ is calculated from (28) where only the observed $y_{i}$ from the last four periods (i.e., 
periods $t-4, t-3, t-2$, and $t-1)$ are included in $\tilde{Y}$. These are the past years' worth of data. No information from period $t$ is included, because if $\rho \neq 0$ in the model from section 4 then that would make $x_{e d}$ an endogenous covariate. For the initial five periods, $\mu$ in the formula is chosen as described above. After that time, $\mu$ instead is taken to be the value of $x_{e d}$ from four periods ago. Since this $\mu$, which incorporates information about all past $y_{i}$ receives as much weight in (28) as any one of the $y_{i}$ from the last four periods, it ensures that old information is downweighted relative to recent information. This formulation of expected regulatory delay can be formalized as

$$
\begin{aligned}
\left(x_{e d}\right)_{t} & =\frac{\sum_{i \in\left\{I_{1}, \ldots, I_{8}\right\}} y_{i}}{\sum_{i \in\left\{I_{1}, \ldots, I_{8}\right\}} d_{i}} \quad \text { for } t=1 \\
\left(x_{e d}\right)_{t} & =\frac{\left(x_{e d}\right)_{1}+\sum_{i \in\left\{I_{t-j-1}, \ldots, I_{t-1}\right\}} y_{i}}{1+\sum_{i \in\left\{I_{t-j-1}, \ldots, I_{t-1}\right\}} d_{i}} \text { for } t=2, \ldots, 5 \\
\left(x_{e d}\right)_{t} & =\frac{\left(x_{e d}\right)_{t-4}+\sum_{i \in\left\{I_{t-4}, \ldots, I_{t-1}\right\}} y_{i}}{1+\sum_{i \in\left\{I_{t-4}, \ldots, I_{t-1}\right\}} d_{i}} \text { for } t \geq 5
\end{aligned}
$$

\section{References}

Ai, Chunrong and Sappington, David E. M. (1998), The Impact of State Incentive Regulation on the U.S. Telecommunications Industry. Mimeo.

Anscombe, F. J. (1950), 'Sampling Theory of the Negative Binomial and Logarithmic Series Distributions', Biometrika 37(3/4), 358-382.

Arrow, Kenneth J. (1962), Economic Welfare and the Allocation of Resources for Invention, in 'The Rate and Direction of Inventive Activity', Princeton, NJ: Princeton University Press.

BellSouth Corporation (1998), 'Comments', Submitted to the FCC., March 27. In the Matter of Computer III Further Remand Proceedings: Bell Operating Company Provision of Enhanced Services.

Braeutigam, Ronald R. (1979), 'The Effect of Uncertainty in Regulatory Delay on the Rate of Innovation', Law and Contemporary Problems 43(3), 98-111. 
Breslow, N. (1974), 'Covariance Analysis of Censored Survival Data', Biometrics 30(1), 89-99.

Bresnahan, Timothy F. (1997), Comment on 'Valuation of New Goods under Perfect and Imperfect Competition', in T. F. Bresnahan and R. J. Gordon, eds, 'The Economics of New Goods', Chicago: University of Chicago Press, pp. 237-247. Studies in Income and Wealth, vol. 58.

Brock, Gerald W. (1994), Telecommunication Policy for the Information Age: From Monopoly to Competition, Cambridge: Harvard University Press.

Cameron, A. Colin and Trivedi, Pravin K. (1998), Regression Analysis of Count Data, Econometric Society Monographs, 30, Cambridge: Cambridge University Press.

Cox, David R. (1975), 'Partial Likelihood', Biometrika 62, 269-276.

Daniel, Joseph I. (1995), 'Congestion Pricing and Capacity of Large Hub Airports: A Bottleneck Model with Stochastic Queues', Econometrica 63, 327-370.

Gilbert, Richard J. and Newbery, David M. G. (1982), 'Preemptive Patenting and the Persistence of Monopoly', American Economic Review 72, 514-526.

Gourieroux, Clement E. and Visser, M. (1997), 'A Count Data Model with Unobserved Heterogeneity', Journal of Econometrics 79, 247-268.

Greenstein, Shane, McMaster, Susan and Spiller, Pablo T. (1995), 'The Effect of Incentive Regulation of Infrastructure Modernization: Local Exchage Companies' Deployment of Digital Technology', Journal of Economics and Management Strategy 4(2), 187-236.

GTE Corporation (1998), 'Comments', Submitted to the FCC., March 27. In the Matter of Computer III Further Remand Proceedings: Bell Operating Company Provision of Enhanced Services.

Hausman, Jerry (1997), 'Valuing the Effect of Regulation on New Services in Telecommunications', Brookings Papers on Economic Activity pp. 1-38. Microeconomics. 
Johnson, Norman L., Kotz, Samuel and Kemp, Adrienne W. (1993), Univariate Discrete Distributions, Wiley Series in Probability and Mathematical Statistics. Probability and Mathematical Statistics, 2nd edn, New York: John Wiley \& Sons.

Joskow, Paul L. and Rose, Nancy L. (1989), The Effects of Economic Regualtion, in R. Schmalensee and R. D. Willig, eds, 'Handbook of Industrial Organization, Vol. 2', North-Holland, Amsterdam, chapter 25, pp. 1449-1506.

Kraushaar, Jonathan M. (1991-1997), 'Fiber Deployment Update'. Industry Analysis Division, Common Carrier Bureau, Federal Communications Commission.

Lancaster, Tony (1990), The Econometric Analysis of Transition Data, Econometric Society Monographs No. 17, Cambridge: Cambridge U. Press.

Mueller, Milton L. (1993), Telephone Companies in Paradise: a Case Study in Telecommunications Deregulation, New Brunswick: Transaction Publishers.

Mulligan, James G. and Hoffman, Saul D. (1998), 'Daycare Quality and Regulation: A QueuingTheoretic Approach', Economics of Education Review 17(1), 1-13.

New Paradigm Resources Group and Connecticut Research (1997), 1997 Annual Report on Local Telecommunications Competition, 8th edn, NPRG.

Pakes, Ariel (1997), 'Valuing the Effect of Regulation on New Services in Telecommunications: Comment', Brookings Papers on Economic Activity pp. 39-54. Microeconomics.

Peterson, Trond (1986), 'Estimating Fully Parametric Hazard Rate Models with Time-Dependent Covariates', Sociological Methods \& Research 14(3), 219-246.

Prieger, James E. (1998), 'The Effects of Regulation on the Innovation and Introduction of New Telecummunications Services', Attachment B to Petition of Ameritech Corporation to Remove 
Barriers to Investment in Advanced Telecommunications Capability, March 5. Submitted to the Federal Communications Commission.

Prieger, James E. (1999), Regulation, Innovation, and the Introduction of New Telecommunications Services, PhD thesis, University of California, Berkeley.

Quandt, Richard E. (1983), Computational Problems and Methods, in Z. Griliches and M. Intriligator, eds, 'Handbook of Econometrics. Volume I', Amsterdam; New York: Elsevier, pp. 699-764.

Ross, Sheldon M. (1983), Stochastic Processes, Wiley Series in Probability and Mathematical Statistics. Probability and Mathematical Statistics., New York: John Wiley \& Sons.

Sappington, David E.M. and Weisman, Dennis L. (1996), 'Potential Pitfalls in Empirical Investigations of the Effects of Incentive Regulation Plans in the Telecommunications Industry', Information Economics and Policy 8, 125-140.

Stine, Robert A. (1985), 'Bootstrap Prediction Intervals for Regression', Journal of the American Statistical Association 80(392), 1026-1031.

Taylor, William E., Zarkadas, Charles J. and Zona, J. Douglas (1992), Incentive Regulation and the Diffusion of New Technology in Telecommunications. NERA mimeo.

United States. Department of Commerce. Bureau of Economic Analysis (1985), An Introduction to National Economic Accounting. Methodology Paper Series MP-1. Washington, DC: GPO.

United States. Federal Communications Commission. Common Carrier Bureau (1986), 'In the Matter of Amendment of Sections 64.702 of the Commission's Rules and Regulations (Third Computer Inquiry), Report and Order', 104 FCC 2d 948.

United States. Federal Communications Commission. Common Carrier Bureau (1987-1997), Statistics of Communications Common Carriers, Washington, D.C.: U.S. GPO. 
United States. Federal Communications Commission. Common Carrier Bureau (1998), 'In the Matter of Computer III Further Remand Proceedings: Bell Operating Company Provision of Enhanced Services, Further Notice of Proposed Rulemaking', 13 FCC Rcd. 6040, January 29.

United States. Federal Communications Commission. Common Carrier Bureau (1999), 'In the Matters of Computer III Further Remand Proceedings: Bell Operating Company Provision of Enhanced Services; and 1998 Biennial Regulatory Review_Review of Computer III and ONA Safeguards and Requirements; CC Docket Nos. 95-20, 98-10, Report and Order', February 24.

Vogelsang, Ingo and Mitchell, Bridger (1997), Telecommunications Competition: The Last Ten Miles, MIT Press, Cambridge.

Winkelmann, Rainer (1995), 'Duration Dependence and Dispersion in Count-Data Models', Journal of Business \& Economic Statistics 13(4), 467-474. 


\section{NOTES}

${ }^{1}$ The Bell Operating Companies are Ameritech, Bell Atlantic, BellSouth, NYNEX, Pacific Bell, Southwestern Bell Telephone, and US West. In most of the study period, these firms were independent. Bell Atlantic merged with NYNEX in 1996 and Southwestern Bell merged with Pacific Bell in 1997; the firms continued to issue separate CEI plans.

${ }^{2}$ BellSouth Comments in the Computer III Further Remand Proceedings, CC Dkt No. 95-20 (1998).

${ }^{3}$ MCI contested Ameritech's CEI plan for Personal Access Service while it added the functionality described in the plan to its competing service. Eventually Ameritech withdrew its plan [FCC Report and Order (1999, footnote 66)].

${ }^{4}$ See Braeutigam (1979) for a model incorporating the first and third effects.

${ }^{5}$ Counts within each category of services are sufficient statistics in a Poisson model that includes only categorical fixed effects. Thus, without knowing the calendar dates of each service only the Poisson fixed effects model presented below is identified, not any of the other innovation models).

${ }^{6}$ Computer III Phase I Order, 104 F.C.C.2d 948 (1986) et al. See Prieger (1999) for the other legal citations for this section.

${ }^{7}$ This section draws from Brock (1994, ch.12) and Vogelsang and Mitchell (1997, ch.6). The FCC eventually relieved AT\&T of most Computer III and ONA requirements.

${ }^{8}$ Services were submitted as early as the first quarter of 1987 . Gateway services are transmissionbased (but not content-based) information services. Content-based services were allowed after July 25, 1991.

${ }^{9}$ An early version of this study was submitted on behalf of Ameritech in the regulatory review proceedings leading up to the February 1999 decision. See Prieger (1998) for the study and FCC Report and Order (1999) for discussion of it in the FCC's decision. 
${ }^{10}$ Queuing theory, developed by engineers and operations researchers, has been applied to economic phenomena by Daniel (1995) and Mulligan and Hoffman (1998).

${ }^{11}$ In particular, if $s_{t}=1, m_{t k \ell} \equiv \exp \left(\boldsymbol{\alpha}_{1 k}+\boldsymbol{\alpha}_{2 l}+\mathbf{x}_{t}^{\prime} \boldsymbol{\beta}\right)$, and $\delta \equiv \exp \left(\tau_{1}^{2}\right)$, then $E\left(n_{t k \ell}\right)=m_{t}$ and $\operatorname{Var}\left(n_{t k \ell}\right)=m_{t}\left[1+m_{t}(\delta-1)\right]($ Anscombe, 1950).

${ }^{12}$ Strictly speaking, if $\rho \neq 0$ then $n_{t k \ell}$ is endogenous and $g_{i}$ is not the true contribution to the joint likelihood if functions of $n_{t k \ell}$ appear as regressors. However, even when a time-varying covariate such as $n_{t k \ell}$ is not exogenous, the expression in (6), and therefore (10), is a partial likelihood (Lancaster, 1990, sec.9.2.11). Because estimates resulting from maximizing a partial likelihood behave exactly as do ML estimates, including consistency of the parameter and s.e. estimates (Lancaster, 1990, sec.9.2.2), I will not further emphasize this distinction.

${ }^{13}$ I use Gaussian quadrature, with 50 evaluation points in both dimensions. See Quandt (1983, section 8.2) for a brief introduction to (and further references for) quadrature.

${ }^{14}$ Even when $\tau_{1}>0$, MLE based on a simple Poisson model yields consistent estimates of $(\boldsymbol{\alpha}, \boldsymbol{\beta})$ as long as the conditional mean is correctly specified (Cameron and Trivedi, 1998, sec. 3.2.3).

${ }^{15}$ At times the FCC requires a BOC to submit a new CEI plan to address concerns it has. There is typically nothing new about the telecommunications service itself in such amendments. Similarly, if a plan was filed because functionality of a service was reduced, this was not counted as a new service. Finally, if two new plans were submitted because two previously distinct technologies had converged, only one of the new plans was counted. See the Appendix to see to which filings these remarks apply.

${ }^{16}$ One change is made to the models in Table 4 when forming the predictions. Since the interim period is neither in the initial Computer III period nor in the subsequent remand period, I do not include the remand indicator in the estimations for any of the predictions. Thus the predictions reflect the average arrival rate in the two CEI periods. Given that the remand coefficient is small in these models, this averaging has little impact on the predictions. 
${ }^{17}$ Only plans and amendments were used in the prediction exercise, both for the prediction and the actual number of service introduced. Waivers were observable during the interim and do not need to be predicted. US West did in fact file a waiver during the interim. I also do not include AT\&T in the prediction exercise because the firm's data ends after 1991.

${ }^{18}$ The prediction interval accounts for variation from two sources: estimation error and the intrinsic variation of the Poisson random variable. The bootstrap prediction interval (adapted from Stine (1985)) covers $95 \%$ of a sample of size 9,999 of $y^{*}+\epsilon_{i}$, where $y^{*}$ is the prediction and $\epsilon_{i}=y_{i}-y_{i}^{*}$ is the prediction error. The $y_{i}$ are pseudo-random deviates drawn from a Poisson distribution with mean $y^{*}$. The $y_{i}^{*}$ are bootstrapped predictions.

${ }^{19} \hat{\tau}_{1}$ is half-normal under the hypothesis that $\tau_{1}=0$, and so the correct $p$-value is based on a one-sided critical value.

${ }^{20}$ The variable measuring expected regulatory delay is constructed assuming the firms believe regulatory delay is exponentially distributed (with rate $\kappa$ ). The expectation is updated via Bayes' rule given observed completed and ongoing delays within the past year. The prior distribution for $\kappa$ is taken to be Gamma, the conjugate prior. See the appendix for details.

${ }^{21}$ The yearly mean of the arrival process is $\lambda_{t k \ell}^{0}$; the elasticity of the mean with respect to $x$ is $\beta x$. When $x=\log (w)$, then the elasticity of the mean with respect to $w$ is $\beta$.

${ }^{22} \mathrm{I}$ also drop the BOC fixed effects because they are highly correlated with the added covariates. The fixed effects capture most of the heterogeneity among BOCs, leaving little variation for the other covariates to explain.

${ }^{23} \mathrm{~A}$ Wald test statistic for the two variables is at the 0.70 quantile of a $\chi^{2}(2)$ distribution. When population is added, it is also insignificant.

${ }^{24}$ The firm R\&D figures are from the annual FCC Statistics of Communications Common Carriers. Industry figures are from National Science Foundation survey data for U.S. firms in the commu- 
nications equipment and electrical components categories. Both are in $\$$ millions, adjusted by the GDP deflator. A Wald test statistic with joint null hypothesis that each coefficient is 0 is at the 0.88 quantile of a $\chi^{2}(2)$ distribution.

${ }^{25}$ The replacement effect (Arrow, 1962) indicates that a monopolist that innovates merely replaces one stream of rents with another, so that the incremental profit from innovation is not as great as for a competitive firm. The efficiency effect (Gilbert and Newbery, 1982) indicates that a new service is worth more to a monopolist than to an entrant who then gains only duopoly profit. Which effect predominates is an empirical matter.

${ }^{26}$ Data are from the FCC Fiber Reports (Kraushaar, 1991-1997) and NPRG (1997).

${ }^{27}$ The variable was constructed by subtracting the BOC's contribution to GSP within its region from the total. The BOC's contribution was estimated as (operating revenue - [operating expenses not including depreciation]) using FCC SOCC data. This approximates the BEA (1985) methodology for calculating gross product.

${ }^{28} \mathrm{~A}$ Wald test statistic with joint null hypothesis that each coefficient is 0 is at the 0.31 quantile of a $\chi^{2}(2)$ distribution.

${ }^{29} \mathrm{~A}$ Wald test statistic is at the 0.51 quantile of a $\chi^{2}(7)$ distribution.

${ }^{30}$ Winkelman (1995) and Gourieroux and Visser (1997) also model count processes with interarrival times. I am not aware of any empirical studies using interarrival times for count data apart from Prieger (1999).

${ }^{31}$ The Cox model uses a partial likelihood method to estimate the effect of regressors on an unspecified baseline hazard. I estimate the baseline hazard via Breslow's (1974) nonparametric method. The prediction is the sum of (interim length ${ }_{i} /$ mean survival time ${ }_{i}$ ) for each firm-plan type. This predictor is the asymptotic mean of the compound counting process generated from the renewal processes associated with the estimated survival functions. The prediction may be biased upward 
due to censoring.

${ }^{32}$ The bootstrap prediction interval covers $95 \%$ of a sample of $y^{*}+\epsilon_{i}$, where $y^{*}$ is the prediction and $\epsilon_{i}=y_{i}-y_{i}^{*}$ is the prediction error. The $y_{i}$ are pseudo-random deviates (1,000,000 draws) drawn from a compound counting process where the interarrival times are drawn from the Cox/Breslow estimated survival curves. The $y_{i}^{*}$ were generated by drawing pseudo-random deviates from the estimated asymptotic distribution of the mean interarrival time and forming the corresponding counts.

${ }^{33}$ In no case does the FCC reject a CEI filing outright. Instead, the FCC notifies the carrier of that to which it objects, and the carrier modifies and resubmits the plan. In such cases the approval delay is calculated from the submission of the first plan to the final approval of the last plan. Withdrawn plans are counted as censored observations.

${ }^{34}$ In the approval delay analysis I exclude the February 1995 en masse filing for the services from the interim. The FCC approved them all at the same time (except for two from Ameritech), indicating an exception to the usual approval process.

${ }^{35}$ These two services have not been approved because of a disagreement between the FCC and the company concerning the classification of an underlying service as basic or enhanced.

${ }^{36} \mathrm{~A}$ me-too filing is a CEI plan that is substantially similar to a previously approved plan filed by another carrier. The FCC rules expedite approval for such plans.

${ }^{37}$ Both a $t$-test (statistic is at the $1.0-3 \mathrm{E}-10$ quantile) and an $L R$ test (statistic is at the $1.0-1 \mathrm{E}-6$ quantile) with $H_{0}: \sigma=1$ and $H_{A}: \sigma \neq 1$ reject the exponential model.

${ }^{38}$ The mean of a Weibull-distributed duration is $\sigma \Gamma(\sigma) \mu_{i}$. When the variables are in logs, the coefficients are elasticities. The percentage change due to an indicator $z$ with coefficient $\delta$ taking a value of 1 is $\% \Delta=\exp (\delta)-1$.

${ }^{39}$ More physical measures of regulatory capacity - FCC total and CEI-related personnel—have little 
variation in the observation period and are not significant when added to the estimations.

${ }^{40}$ These are both treated as time-varying covariates, as explained in section 4 . The former is calculated as the number of unapproved filings at the time of the start of the split duration. The latter is calculated as $\sum_{k \in K} \sum_{\ell \in L} n_{t k \ell}$ for the period of the split duration.

${ }^{41}$ Figure 3 suggests two reasons the result might be spurious. First, there are a large number of services in process during 1995 due to the en masse filing after the remand, which may have undue influence. However, dummying out this period barely changes the coefficients on the two congestion variables. Second, during the remand period (after 1995), there are generally more services in process. Again, dummying the remand period hardly changes the coefficients.

${ }^{42}$ There is a sample selection issue with the covariates estimation. FCC Record pages and the number of commenters are available only for observations that appear in the Record. This necessarily excludes all plans that were withdrawn by the carriers and those for which approval had not yet been granted by the end of the observation period (14 observations). These observations may not be a random subsample because plans with the longest expected delay are most likely to be withdrawn or censored. This may account for the shorter predicted mean from the covariates estimation. The impact of sample selection bias is not likely to be severe; none of the coefficients included in both the congestion and the covariates estimations changes sign.

${ }^{43}$ Finding the services introduced requires a result from queuing theory. If arrivals are Poisson with rate $\lambda$ and the $\mathrm{CDF}$ of the delays is $G$, then the services introduced (i.e., the output of the infinite server queuing system) at time $t$ is a nonhomogeneous Poisson process with mean $\Lambda(t)=\lambda \int_{0}^{t} G(s) d s$ (Ross, 1983, p.39). The actual formula used is a modification of this result reflecting two extensions: the arrival process in Scenario 1 is generalized Poisson, and the arrival rate varies over the observation period because of the heterogeneity and the remand indicator. Details of the calculations in this section are in an appendix.

${ }^{44}$ The standard deviations in the table account for intrinsic (not estimation) variation. The dis- 
tributions of the projected services introduced random variables are not Gaussian, but the usual confidence interval based on twice the s.d. will have approximately correct coverage.

${ }^{45}$ This is a property of any ergodic infinite server queuing system.

${ }^{46}$ See comments by Pakes (1997); see also Bresnahan (1997) for comments on another application of the same econometric methodology.

${ }^{47}$ Three is the 0.13 quantile of a Poisson distribution with mean 6.3, the estimated mean for that time period from the fixed effects model ; no reasonably sized test would reject the hypothesis that the mean of the arrival process was constant during that time.

${ }^{48}$ Vogelsang and Mitchell (1997), ch.6; GTE (1998) comments in the Computer III Further Remand Proceedings also state that the number of requests for interconnection was "modest."

${ }^{49}$ FCC, Computer III Further Remand Proceedings FNPRM (January 29, 1998) at 64.

${ }^{50}$ Without the possibility of the FCC requiring changes to the CEI plan before the service is available, the firm's expected costs of plan preparation drops. 


\begin{tabular}{|c|c|c|c|c|}
\hline Carrier & $\begin{array}{c}\text { Initial } \\
\text { CEI Regime } \\
(\mathbf{1 9 8 7}-1992 / 3)\end{array}$ & $\begin{array}{c}\text { Structural } \\
\text { Separation Lifted } \\
(1992 / 3- \\
\text { Jan 1995) } \\
\end{array}$ & $\begin{array}{l}\text { Remand- } \\
\text { Back to CEI } \\
\text { Regime } \\
(1995-1997)\end{array}$ & Total \\
\hline Ameritech & 2 & 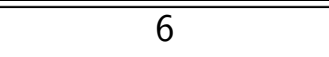 & $\overline{66}$ & 14 \\
\hline Bell Atlantic & 11 & 1 & 4 & 16 \\
\hline BellSouth & 7 & 2 & 2 & 11 \\
\hline NYNEX & 3 & 2 & 3 & 8 \\
\hline PacBell & 8 & 6 & 0 & 14 \\
\hline SWBT & 4 & 3 & 8 & 15 \\
\hline U S West & 4 & $7^{\dagger}$ & 1 & 12 \\
\hline AT\& $T^{*}$ & 11 & - & - & 11 \\
\hline Total & 50 & 27 & $24^{\ddagger}$ & 101 \\
\hline
\end{tabular}

* After 1991, the FCC treated enhanced services from AT\&T differently than those from the BOCs. To preserve comparability, new services by AT\&T after 1991 are not included in the data set.

$\dagger$ Includes a waiver filed during the interim.

‡ Excludes an FCC-mandated payphone plan for each BOC in 1997. The services were not new.

Table 2: New Telecommunications Information Services Introduced Through CEI Plans, Amendments, and Waivers 


\begin{tabular}{ccccccc}
\hline $\begin{array}{c}\text { Obser- } \\
\text { vations }\end{array}$ & Min. & $\begin{array}{c}\text { First } \\
\text { Quartile }\end{array}$ & Median & Mean & $\begin{array}{c}\text { Third } \\
\text { Quartile }\end{array}$ & Max. \\
\hline \hline 784 & 0.00 & 0.00 & 0.00 & 0.10 & 0.00 & 3.04 \\
\hline
\end{tabular}

Table notes: units of observation are $n_{t k \ell}$, the number of new CEI filings from a given firm and of a given type (filings, amendments, waivers) per quarter. When less than the entire quarter is observed, the count is extrapolated to the entire quarter for purposes of these statistics.

Table 3: Summary Statistics for the Innovation Count Data

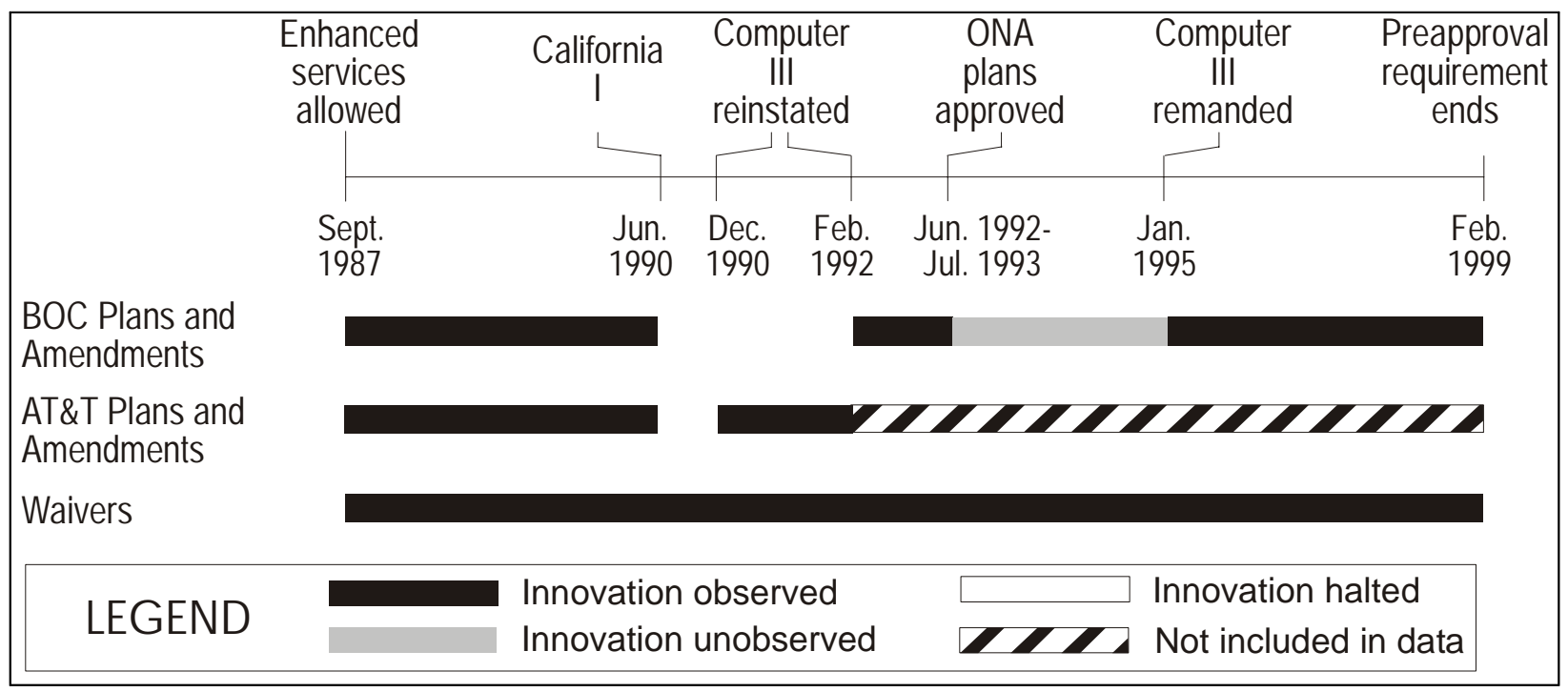

Figure 1: Time Line for the Comparably Efficient Interconnection Regime 


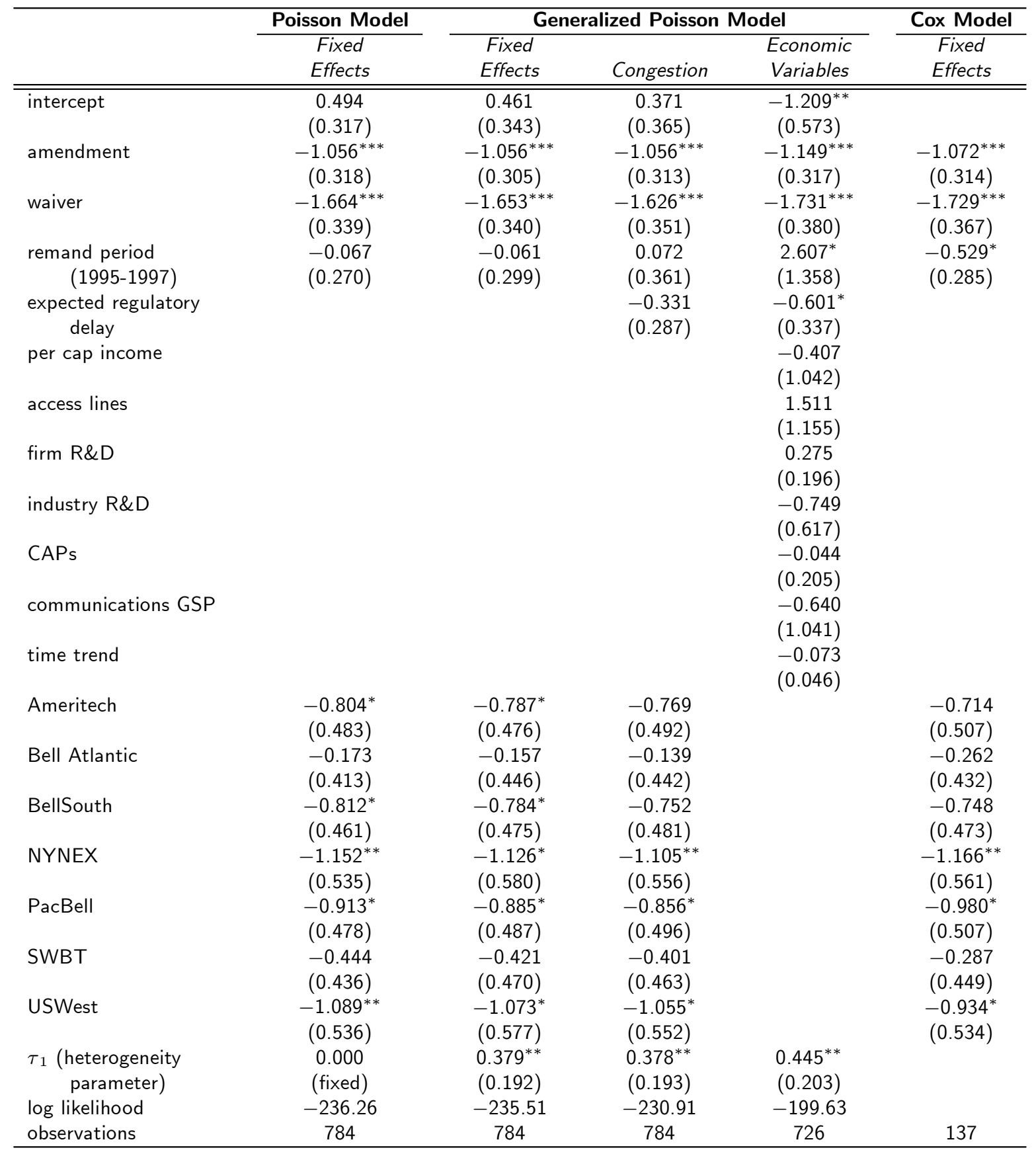

$*=10 \%$ level significance; ${ }^{* *}=5 \%$ level significance; $* * *=1 \%$ level significance; stars for $\tau_{1}$ based on one-sided test. Table notes: Asy. s.e. in parentheses. Dependent variable is the number of new CEI filings of a given type per quarter except for the Cox model, for which it is the interarrival times of the filings. The conditional mean of the dependent variable is exponential-linear in the covariates. Excluded carrier dummy is AT\&T. All covariates are in logs, and adjusted by GDP deflator where appropriate. Income data are from BEA REIS, firm R\&D data from the FCC Statistics of Communications Common Carriers, industry R\&D data from the NSF R\&D in Industry, Competitive Access Providers (CAPs) from NPRG Local Telecom Competition Report and FCC Fiber Deployment Update, and communications industry GSP (net of BOC contributions) from BEA Gross Product by Industry.

\section{Table 4: Estimation Results for the Innovation Model (Independent Version)}




\begin{tabular}{lcccc}
\hline Carrier & $\begin{array}{c}\text { Estimated Yearly } \\
\text { New Plans and } \\
\text { Amendments* }\end{array}$ & $\begin{array}{c}\text { Length of } \\
\text { Interim } \\
\text { (in years) }\end{array}$ & $\begin{array}{c}\text { Predicted Number of } \\
\text { New Services* } \\
\text { During Interim }\end{array}$ & $\begin{array}{c}\text { Actual Number of } \\
\text { New Services* } \\
\text { During Interim }\end{array}$ \\
\hline \hline Ameritech & 0.96 & 2.57 & 2.47 & 6 \\
Bell Atlantic & 1.81 & 2.59 & 4.68 & 1 \\
BellSouth & 0.96 & 1.49 & 1.43 & 2 \\
NYNEX & 0.68 & 2.07 & 1.41 & 2 \\
PacBell & 0.87 & 1.64 & 1.42 & 6 \\
SWBT & 1.39 & 2.19 & 3.02 & 3 \\
U S West & 0.72 & 2.59 & 1.87 & $6^{*}$ \\
Total & 7.39 & & 16.30 & $26^{*}$ \\
\hline
\end{tabular}

*Excludes waivers. Waiver requests were permitted and observed during the interim, and thus do not need to be predicted.

Table note: predictions are based on the Poisson fixed effects model estimated with data from the regulated era. A prediction that differs from the actual number of services created is evidence that innovation changed when the firms were released from regulation.

Table 5: Predicted vs. Actual Innovation of Information Services During the Interim Period

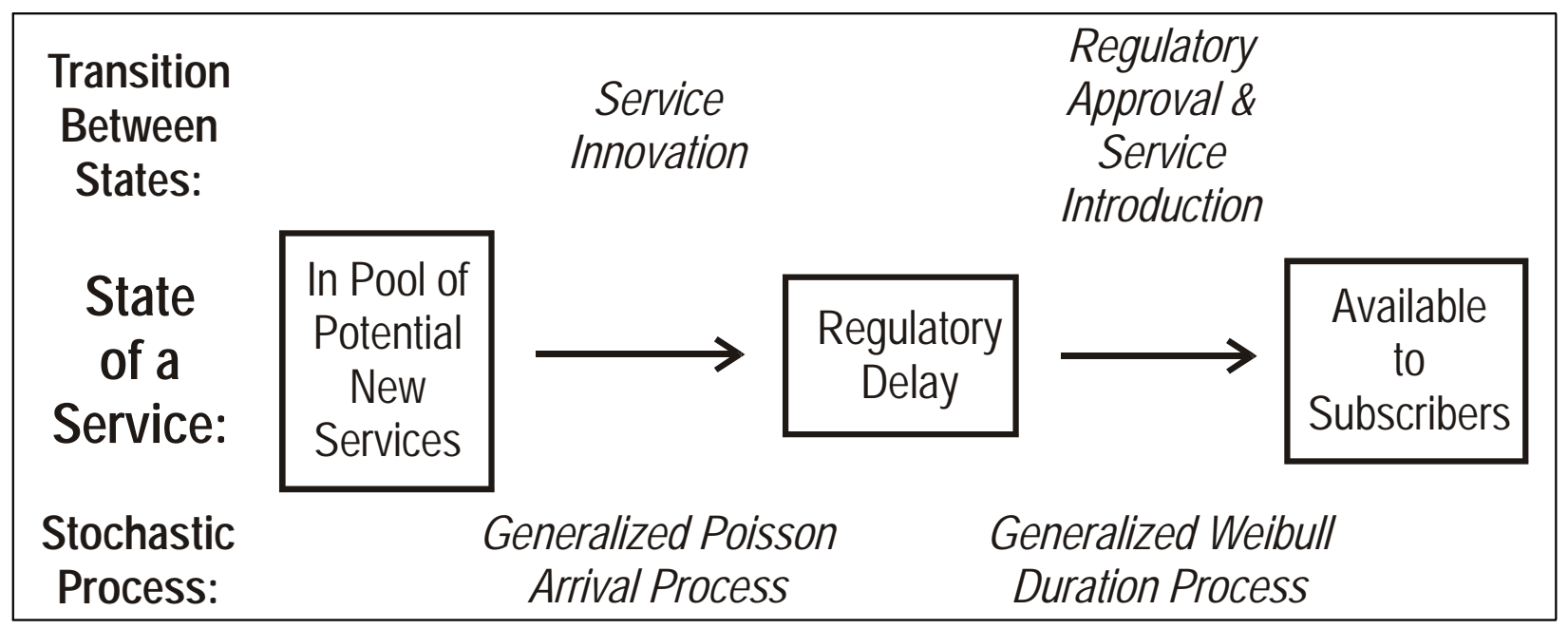

Figure 2: The Innovation and Regulatory Delay Model 


\begin{tabular}{lccc}
\hline & $\begin{array}{c}\text { Prediction* }^{*} \\
\text { (Assuming No } \\
\text { Structural Change) }\end{array}$ & $\begin{array}{c}95 \% \\
\text { Prediction } \\
\text { Interval }^{\dagger}\end{array}$ & $\begin{array}{c}\text { P-value of } \\
\text { Actual Number of } \\
\text { Interim Services }^{\ddagger}\end{array}$ \\
\hline \hline $\begin{array}{l}\text { Poisson Model } \\
\text { Generalized Poisson Model }\end{array}$ & 16.30 & $(7.5,25.6)$ & 0.0197 \\
$\quad$ Fixed Effects & 16.13 & $(7.6,25.8)$ & 0.0225 \\
$\quad$ Congestion & 13.35 & $(5.7,21.9)$ & 0.0024 \\
$\quad$ Economic Variables & 13.09 & $(6.4,22.9)$ & 0.0048 \\
Cox Model & 15.01 & $(7.8,23.1)$ & 0.0048 \\
\hline
\end{tabular}

*Excludes waivers. Waiver requests were permitted and observed during the interim, and thus do not need to be predicted.

†95\% centered prediction interval based on bootstrap prediction errors (see notes 18 and 32).

$\ddagger$ One-sided $p$-value of 26 services.

Table notes: predictions calculated using actual covariates during interim period.

Table 6: Predictions and Prediction Intervals for the Innovation of Information Services During the Interim Period

\begin{tabular}{|c|c|c|c|c|c|c|c|}
\hline & $\begin{array}{c}\text { Obser- } \\
\text { vations }\end{array}$ & Min. & $\begin{array}{c}\text { First } \\
\text { Quartile }\end{array}$ & Median & Mean & $\begin{array}{c}\text { Third } \\
\text { Quartile }\end{array}$ & Max. \\
\hline \multicolumn{8}{|l|}{ 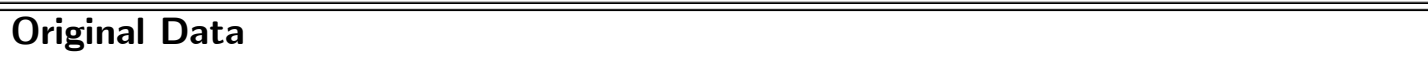 } \\
\hline complete spells & 68 & 0.13 & 0.34 & 0.49 & 0.56 & 0.63 & 1.82 \\
\hline censored spells & 14 & 0.12 & 0.34 & 0.47 & 0.66 & 0.71 & 1.76 \\
\hline all spells & 82 & 0.12 & 0.34 & 0.49 & 0.57 & 0.65 & 1.82 \\
\hline \multicolumn{8}{|l|}{$\begin{array}{l}\text { Modified Data } \\
\text { (split for straddles) }\end{array}$} \\
\hline all spells & 245 & 0.00 & 0.11 & 0.21 & 0.18 & 0.25 & 0.25 \\
\hline
\end{tabular}

Table notes: units of observation are $y_{m}$ for the original data and $y_{m j}-y_{m(j-1)}$ for the modified data, in years. See Section 4 for variable definitions and details on splitting straddling observations.

Table 7: Summary Statistics for the Regulatory Approval Delay Data 


\begin{tabular}{|c|c|c|c|c|}
\hline \multirow[b]{2}{*}{ Variable } & \multirow{2}{*}{$\begin{array}{c}\text { Weibull Model } \\
\text { Fixed Effects }\end{array}$} & \multicolumn{3}{|c|}{ Generalized Weibull Model } \\
\hline & & Fixed Effects & Congestion & Covariates \\
\hline \multirow[t]{2}{*}{$\overline{\text { intercept }}$} & $-0.655^{* * *}$ & -0.403 & -0.349 & $-0.464^{* *}$ \\
\hline & $(0.239)$ & $(0.303)$ & $(0.280)$ & $(0.228)$ \\
\hline \multirow[t]{2}{*}{ BOC (not AT\&T) } & 0.318 & 0.242 & 0.288 & 0.041 \\
\hline & $(0.251)$ & $(0.257)$ & $(0.257)$ & $(0.227)$ \\
\hline \multirow[t]{2}{*}{ amendment } & -0.095 & -0.341 & $-0.437^{*}$ & $-0.674^{* * *}$ \\
\hline & $(0.249)$ & $(0.290)$ & $(0.258)$ & $(0.231)$ \\
\hline \multirow{2}{*}{ waiver } & 0.144 & 0.044 & 0.017 & 0.262 \\
\hline & $(0.238)$ & $(0.255)$ & $(0.240)$ & $(0.195)$ \\
\hline \multirow[t]{2}{*}{ me-too filing } & $-0.522^{* *}$ & $-0.504^{* *}$ & $-0.442 *$ & $-0.363^{* *}$ \\
\hline & $(0.229)$ & $(0.238)$ & $(0.231)$ & $(0.177)$ \\
\hline \multirow[t]{2}{*}{ refiled } & 0.389 & 0.354 & 0.357 & 0.255 \\
\hline & $(0.332)$ & $(0.302)$ & $(0.299)$ & $(0.248)$ \\
\hline filings awaiting approval & & & -0.107 & -0.131 \\
\hline at start of spell & & & $(0.140)$ & $(0.113)$ \\
\hline filings arriving during & & & $-0.288^{* *}$ & -0.133 \\
\hline spell & & & $(0.119)$ & $(0.102)$ \\
\hline pages in FCC Record & & & & 0.123 \\
\hline (in logs) & & & & $(0.104)$ \\
\hline commenters & & & & $0.046^{*}$ \\
\hline (in logs) & & & & $(0.025)$ \\
\hline$\sigma$ (Weibull & $0.613^{* * *}$ & $0.570^{* * *}$ & $0.554^{* * *}$ & $0.426^{* * *}$ \\
\hline shape parameter) & $(0.060)$ & $(0.067)$ & $(0.065)$ & $(0.049)$ \\
\hline$\tau_{2}$ (heterogeneity & 0.000 & $0.323^{* * *}$ & $0.235^{* *}$ & 0.103 \\
\hline parameter) & (fixed) & $(0.131)$ & $(0.128)$ & $(0.129)$ \\
\hline $\begin{array}{l}\text { predicted mean duration (years) } \\
\quad \text { (ave. in sample) }\end{array}$ & 0.620 & 0.703 & 0.757 & 0.585 \\
\hline log likelihood & -19.79 & -18.59 & -16.30 & 7.34 \\
\hline observations & 245 & 245 & 245 & 189 \\
\hline
\end{tabular}

$*=10 \%$ level significance; ${ }^{* *}=5 \%$ level significance; ${ }^{* * *}=1 \%$ level significance; stars for $\sigma$ and $\tau_{2}$ based on one-sided test.

Table notes: asy. s.e. in parentheses. The conditional mean of log dependent variable is linear in the regressors.

Table 8: Estimation Results for the Regulatory Approval Delay Model (Independent Version) 


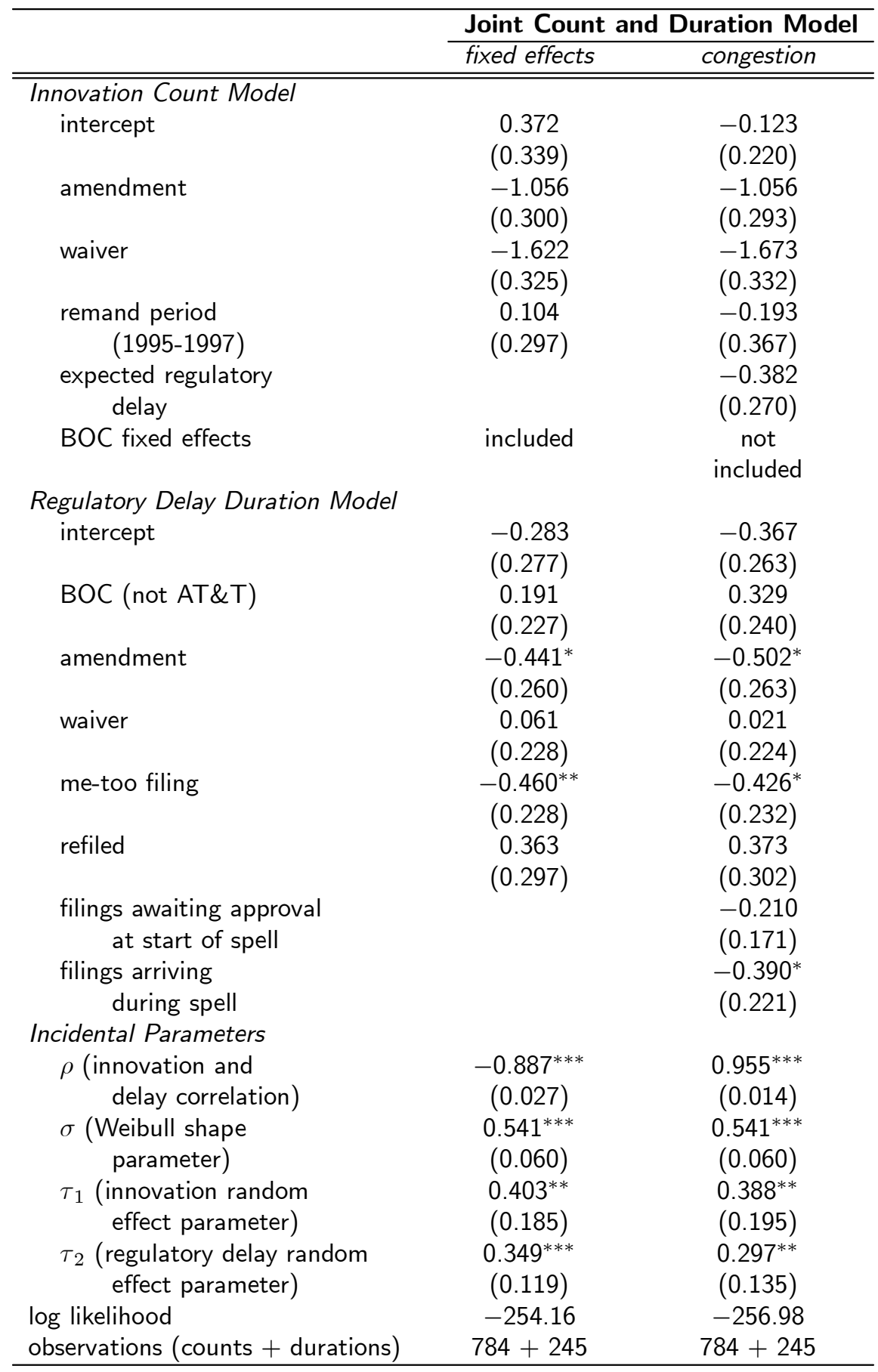

$*=10 \%$ level significance; ${ }^{* *}=5 \%$ level significance; ${ }^{* * *}=1 \%$ level significance; stars for $\sigma, \tau_{1}$, and $\tau_{2}$ based on one-sided test.

Table notes: asy. s.e. in parentheses. See notes to Tables 4 and 8.

Table 9: Estimation Results for the Full Model (Joint Version) 


\begin{tabular}{lccc}
\hline & Scenario 1: & Scenario 2: & \\
& CEI Regime Conditions & Interim Conditions & Difference \\
\hline \hline Observation Period (years) & 11.00 & 11.00 & \\
Projected Services Created & 92.46 & 140.30 & \\
Projected Services Not Approved & 5.74 & 0.00 & \\
Projected Services Introduced & 86.72 & 140.30 & 53.58 \\
$\quad$ standard deviation & 10.69 & 11.84 & 15.96 \\
\hline
\end{tabular}

Table notes: Services Not Approved are arrivals still awaiting regulatory approval at end of period. See text and notes 43,44 for details.

Table 10: Projections for the Innovation and Introduction of Information Services During the Entire Observation Period

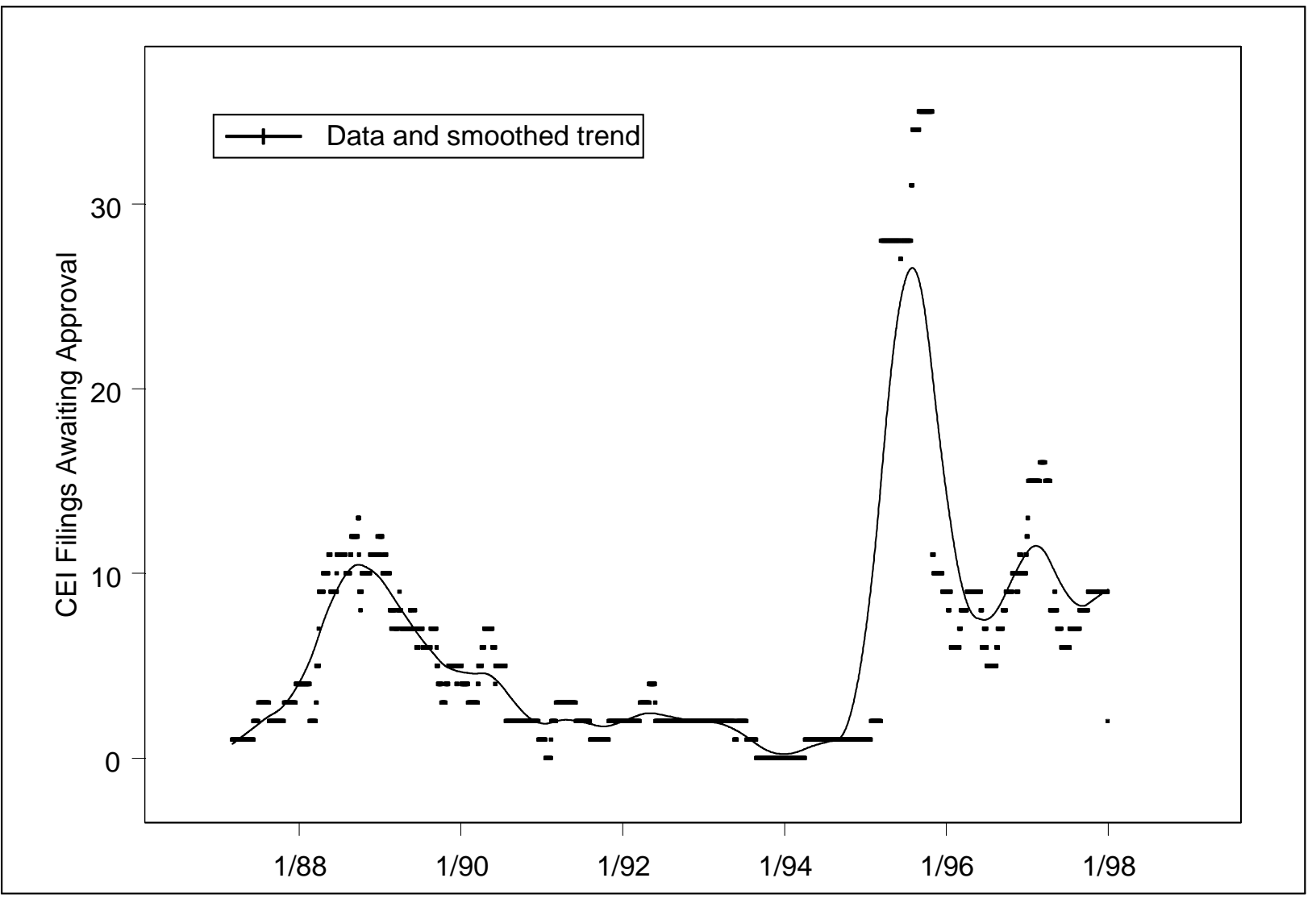

Figure 3: CEI Filings Awaiting Regulatory Approval 